\title{
An analysis of top author citations in software engineering and a comparison with other fields
}

\author{
Kai Petersen ${ }^{1,2}$ (D) Nauman Bin $\mathrm{Ali}^{2}$
}

Received: 16 March 2021 / Accepted: 20 August 2021 / Published online: 9 October 2021

(c) The Author(s) 2021

\begin{abstract}
Ioannidis et al. provided a science-wide database of author citations. The data offers an opportunity to researchers in a field to compare the citation behavior of their field with others. In this paper, we conduct a systematic analysis of citations describing the situation in software engineering and compare it with the fields included in the data provided by Ioannidis et al. For comparison, we take the measures used by Ioannidis into consideration. We also report the top-scientists and investigate software engineering researchers' activities in other fields. The data was obtained and provided by Ioannidis et al. based on the Scopus database. Our method for analysis focuses on descriptive statistics. We compared software engineering with other fields and reported demographic information for the top authors. The analysis was done without any modifications to the ranking. In the later analysis, we observed that $37 \%$ of researchers listed as software engineers were not in the software engineering field. On the other hand, the database included a large portion of top authors (ca. $60 \%$ to $80 \%$ ) identified in other software engineering rankings. Other fields using the database are advised to review the author lists for their fields. Our research's main risk was that researchers are listed that do not belong to our studied field.
\end{abstract}

Keywords Software engineering $\cdot$ Author citations $\cdot$ Self-citations $\cdot$ Comparison

\section{Introduction}

Scientific measures are collected on various levels, for example, to assess institutions (Karanatsiou et al. 2019), scientific publication forums (Pendlebury and Adams 2012), impact of particular publications (Garousi and Fernandes 2016), and individual researchers (Karanatsiou et al. 2019),(Karanatsiou et al. 2019). The rankings in software engineering investigated the contributions of researchers using different measures.

The most prominent ranking for software engineering researchers was done for a series of time intervals (e.g., Glass and Chen 2003 for the time period 1998-2002) and was published by the Journal of Systems and Software (JSS). The measure used was based on

Kai Petersen

kai.petersen@bth.se

1 University of Applied Sciences, Flensburg, Germany

2 Blekinge Institute of Technology, Karlskrona, Sweden 
publications in the most influential journals of the field. Also, depending on author configurations (number of authors and positioning), a journal publication was counted differently (e.g., single-author papers were weighted higher). For the most recent edition of the JSS ranking, the counting rules and measures were changed. For example, the new ranking included more publication forums, and author citations were considered. Another recent assessment of top authors in software engineering was presented by Fernandes (2014). Fernandes used two different measures to rank the researchers: fractional and harmonic authorship credit (cf. Hagan Hagen 2014). Just considering the software engineering field, different rankings use different measures and obtain the basis for ranking (selected publication forums) in different ways.

Ioannidis et al. (2020) provided a science-wide database containing the scientific measures for top authors. The measure used for the ranking was referred to as Comosite Citation index, which were introduced in an earlier publication by Ioannidis et al. (2016). The Composite Citations index combines different measures of author performance into a single measure. The database covers 21 research fields and also documents subfields within the fields (in total 174) ${ }^{1}$. Each researcher was classified according to fields and subfields. The database provides the opportunity to add to the previous rankings, as the same measures were used across different fields and sub-fields. Therefore, a comparison of fields and subfields becomes possible. Furthermore, previous software engineering rankings emerged from rankings done by other software engineering researchers. As a non-software engineering researcher constructed the database, the risk of unintentional biases is reduced.

This paper's main contribution is to provide top authors in software engineering based on the database provided by Ioannidis et al. More specifically, we make the following contributions:

- C1: Compare software engineering with other fields in the database. Given that Ioannidis et al. use the same measures across fields, a unique opportunity is provided, allowing for such a comparison.

- C2: Characterize the top authors in software engineering based on the measures provided. We investigate different measures, such as top countries in terms of the number of scholars ranked, top universities, as well as age profile and research productivity. We also investigate which other sub-fields (besides software engineering) are associated with the top authors.

It is essential to highlight that we first analyze the data as-is. That is, no modifications are done to the data-set not to introduce bias in the initial analysis.

After that, we review the data in detail concerning validity issues concerning the data for software engineers. We also compare the findings from analyzing the database with the results of previous software engineering rankings and reflect on the measures used. Consequently, our analysis provides insights to other fields that use the database by Ioannidis et al. for analysis of their fields.

Section 2 (Related work) presents previous rankings of software engineering authors. Section 3 describes the data collection and resulting database and the variables studied. Section 4 presents the results. In Sect. 5 we compare the rankings and discuss the measures used. Section 6 concludes the paper.

\footnotetext{
${ }^{1}$ The data file (Table-S8-Field-Subfield-Thresholds-career-2019.xlsx) can be found in Mendeley at https:// dx.doi.org/10.17632/btchxktzyw
} 


\section{Related work}

Several studies have used bibliometrics data to reflect on various aspects of software engineering (SE) research.

Mathew et al. (2019) used topic modeling and bibliometric data (author names, venues, and citations) to identify ten major topics in SE research. Their study showed the changes in the major topics over the years as well. They also confirmed the higher citation count of journal articles compared to conference papers. They also investigated whether there is a gender gap in the authorship of publications on the top topics.

Fernandes (2014) reviewed proceedings and volumes of 122 conferences and journals and identified that the number of co-authors in SE publications is increasing over the years. The results were re-validated in another study by Fernandes and Monteiro in another study (Fernandes and Monteiro 2017). The findings are useful as they highlight a positive trend indicating more collaborative research, which is necessary for dealing with complex real-world challenges. Furthermore, the results highlight the need for clear guidelines for being co-authors and describing the role and contribution of the coauthors in the study.

In a series of 15 articles published from 1994 to 2011, researchers have published a ranking of most productive scholars and institutions in systems and software engineering (Glass 1994; Wong et al. 2011). In these studies, they have relied on the number of papers published in a few key SE journals and conferences by authors in a sliding 5-year period.

Garousi and Fernandes (2016) identified the top 100 most cited papers in SE research among approximately 70,000 papers. They further noticed that about $43 \%$ of the papers had no citations at all (Garousi and Fernandes 2017). Garousi and Fernandes (2017) investigated if there are differences in citation patterns for paper publication venues and the authors' countries.

In our previous work, we have looked at citation behavior in SE literature. Molléri et al. (2018) analyzed if the number of citations to a publication is related to its reporting quality. They found some association between the reported rigor of the empirical study and the number of citations. Poulding et al. (2015) identified that the number of citations alone is insufficient for assessing the academic impact of a publication. They evaluated using a citation behavior taxonomy (Bornmann and Daniel 2008) to classify and analyze citations to highly cited papers in leading SE conference proceedings. In another study, Ali et al. (2020) similarly used another classification scheme (Teufel et al. 2006) to reflect on the nature and quality of citations.

To the best of our knowledge, the citation behavior in SE research and its comparison to other fields has not been investigated yet. This study fills this gap by examining and comparing the SE researchers' profile, academic impact, and engagement in multiple fields with other scientific fields.

\section{Method}

Our study was motivated by the possibility to see how distributions of citations differ between other fields and software engineering, which was enabled by the database provided by Ioannidis et al. (2020). 


\section{Research questions}

We formulated three research questions, one question focusing on the comparative analysis (Contribution C1), and two focusing on a more detailed analysis of the top authors in software engineering (Contribution C2). We argue that it is interesting to look at this ranking compared to other rankings, as software engineering researchers have not collected the data, and the measures were also defined independently, which merits a new analysis of top scientists.

- Research question 1 (RQ1,C1): How do author citations in software engineering compare to other fields concerning the citation measures provided by Ioannidis et al.?

- Research question $2(R Q 2, C 2)$ : What are the demographics of the top authors (e.g., country of origin, institutions, etc.)?

- Research question 3 (RQ3, C2): In which other fields software engineering authors are active?

\section{Data collection}

Ioannidis et al. extracted the data using researcher profiles from the Scopus database. The extraction focused on author profiles. Overall a total of 7.92 million author profiles were extracted. The standardized citation data used in this study was created by Ioannidis et al. (2020) by collecting raw citation data from Scopus on May 6, 2020. Ioannidis et al. included the top $100 \mathrm{k}$, and also authors were considered that belong to the top $2 \%$ in their subfield discipline, and otherwise would not have made it onto the list.

The focus was on career-long impact. That is, the study considered the statistics for the years 1960 to the end of 2019. The authors also continuously update the database for future analysis.

The ranking used the Composite Citation index, which is derived measure from multiple indicators, and described in the following section. It is also noteworthy that Ioannidis et al. removed self-citations for the ranking. They found that this impacted the ranking, as $4.9 \%$ of scientists in the list with self-citations are not on the list without self-citations.

\section{Analysis}

A range of measures has been presented based on each researcher's profile. Table 1 lists and defines the measures. While most measures are self-explanatory (e.g., np6019) or generally well known (e.g., h-index), Composite Citation index and the definition of fields and sub-fields are interesting to discuss in further depth.

Composite citation index: Ioannidis et al. (2016) recognizing the increasing trend of multi-authorship, argued for the need of a Composite Citation index that considers multiple indicators to assess a researcher's impact. They (Ioannidis et al. 2016) considered nine indicators but based on a correlation analysis selected the following six to comprise the Composite Citation index $c$ :

$n c: \quad$ Total citations. 


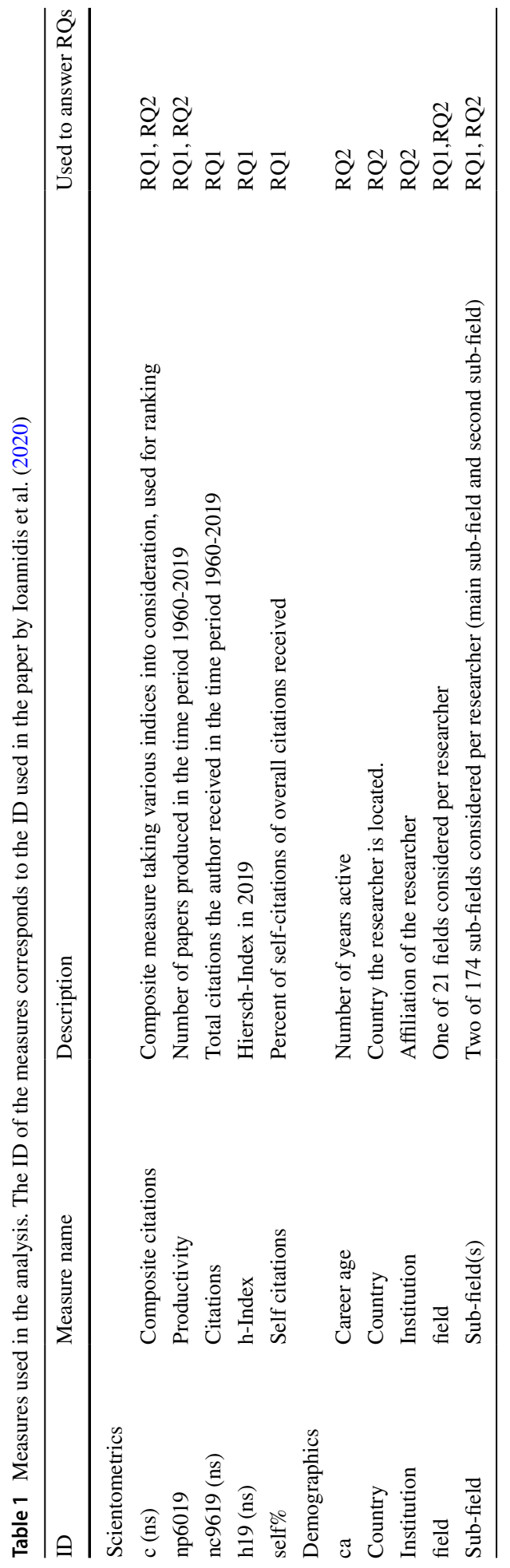


$h$ : Hirsch $\mathrm{h}$-index, which is the maximum value $\mathrm{h}$ when $\mathrm{h}$ papers of an author have been cited at least $\mathrm{h}$ times.

hm: $\quad$ Schreiber Hm index, which provides co-author adjustment to h-index (Schreiber 2008).

ncs: $\quad$ total citations to sole-authored papers

ncsf: $\quad$ total citations to papers for which the researcher is the sole or the first author.

$n c s f$ : total citations to papers for which the researcher is the sole, first, or the last author.

The above six indicators are log-transformed to a value between 0 and 1 . These values are summed together to derive the Composite Citation index C (Ioannidis et al. 2016).

To avoid introducing bias as software engineering researchers (analyzing the data for our field), we report top-scientists (top $2 \%$ ) fully ${ }^{2}$.

Comparative analysis (RQ1): To answer the first research question (RQ1), we used descriptive statistics. We compared the distribution of the different measures of software engineering to other fields. An alternative could have been to compare with all 174 subfields. However, this would have resulted in too many comparisons to comprehend the data easily. However, we analyzed the number of the 172 sub-fields being ranked above or below the median, minimum, and maximum values for software engineering.

Top software engineering authors (RQ2/RQ3): When analysing the top authors in software engineering, we used simple frequency analysis to rank the countries and institutions based on the number of top authors associated with them. The measures of career age and productivity are not included in the Composite Citation index. Thus, we investigated the association between the two measures. We also checked the correlation using Kendall's $\tau$, which is more robust compared to other correlation measures when the data sets have outliers (Croux and Dehon 2010).

\section{Results}

The results present the answers to the research questions, namely comparison with other fields (RQ1) and after that the detailed analysis of top authors in software engineering (RQ2).

\section{RQ1: comparison with other fields}

As described earlier, the authors were assigned to fields and sub-fields. Concerning the field "Information \& Communication Technologies (ICT)", we compared the ICT subfield software engineering with other subfields in ICT. Here, we focus on the comparison of the Composite Citation index. Later, we compare software engineering with the overall ICT category regarding the other measures (see Table 1).

\footnotetext{
${ }^{2}$ The standardized data by Ioannidis et al. can be found on Mendeley at https://dx.doi.org/10.17632/btchx ktzyw
} 
Boxplot grouped by sm-subfield-1

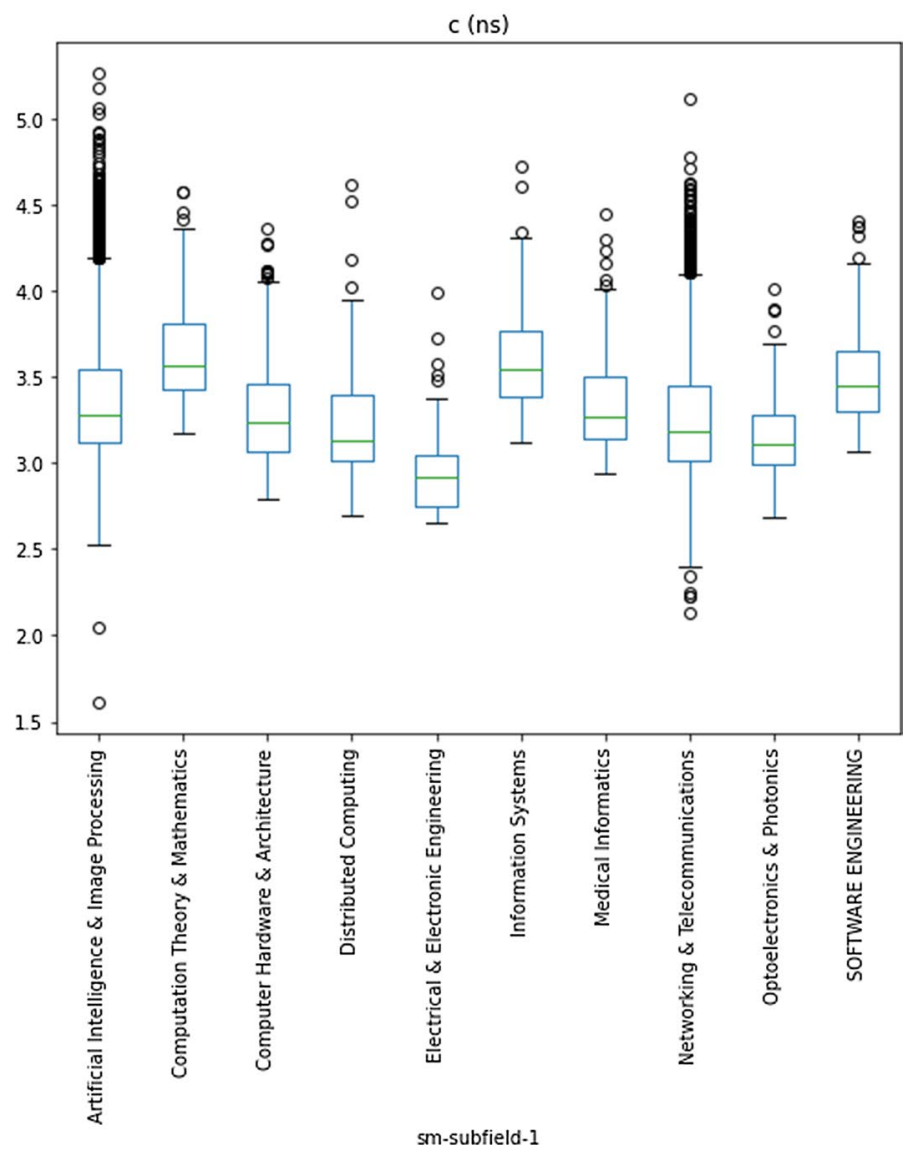

Fig. 1 Comparison of software engineering with sub-fields in the field "Information \& Communication Technologies"

\section{Comparison of software engineering with other ICT subfields}

Figure 1 shows the distribution of the Composite Citation index of software engineering (SE) and other subfields in the ICT category. Only two fields have higher median values than SE, namely "Computational Theory \& Mathematics" and "Information Systems". It is also noteworthy that the fields "Networking \& Telecommunications" and "Artificial Intelligence \& Image Processing" have a higher number data points (researchers) that are outliers concerning their Composite Citation index.

\section{Comparison of software engineering with other fields}

Figure 2 shows the comparison of software engineering with other fields concerning the distribution of the studied measures (see Table 1). Table 2 shows the ranking of software engineering and the other fields based on medians. 


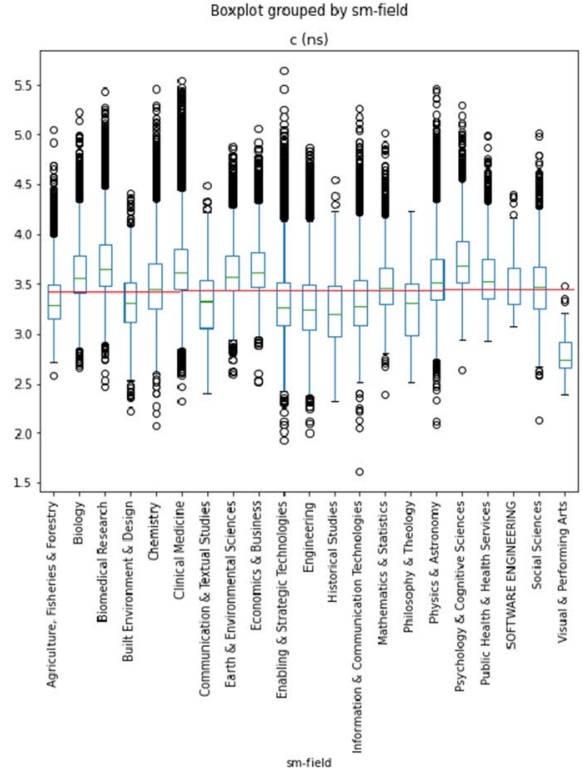

(a) c (ns) - Composite Citation index

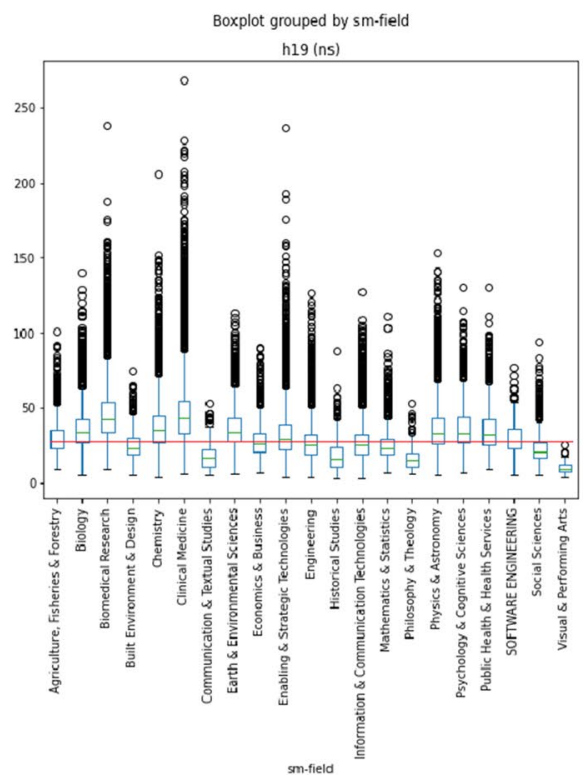

(c) h19 (ns) - H-Index (no self-citations)

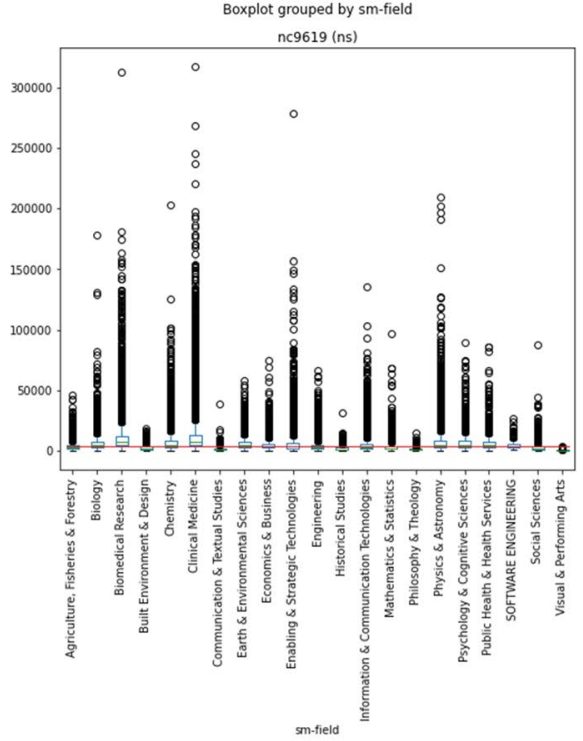

(b) nc9619 (ns) - Citations 1960-2019 (no self-citations)

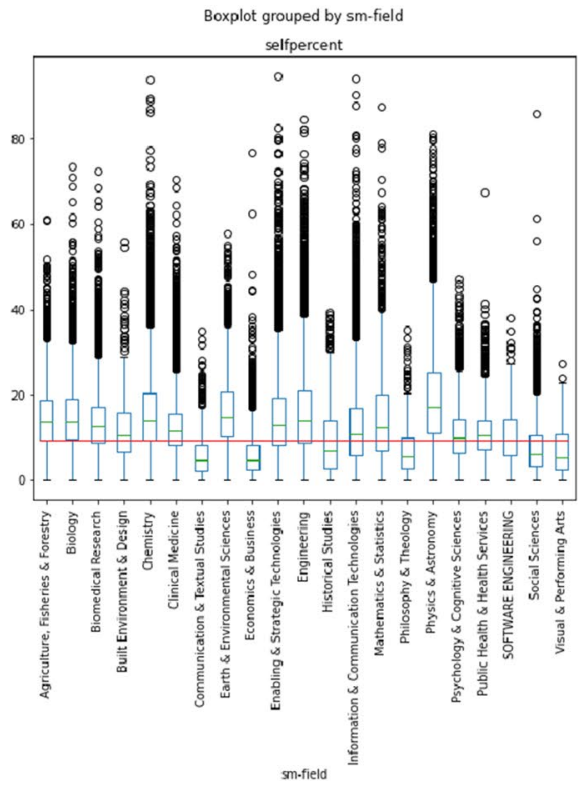

(d) Percent of self-citations

Fig. 2 Data distributions for software engineering and comparison with other fields 


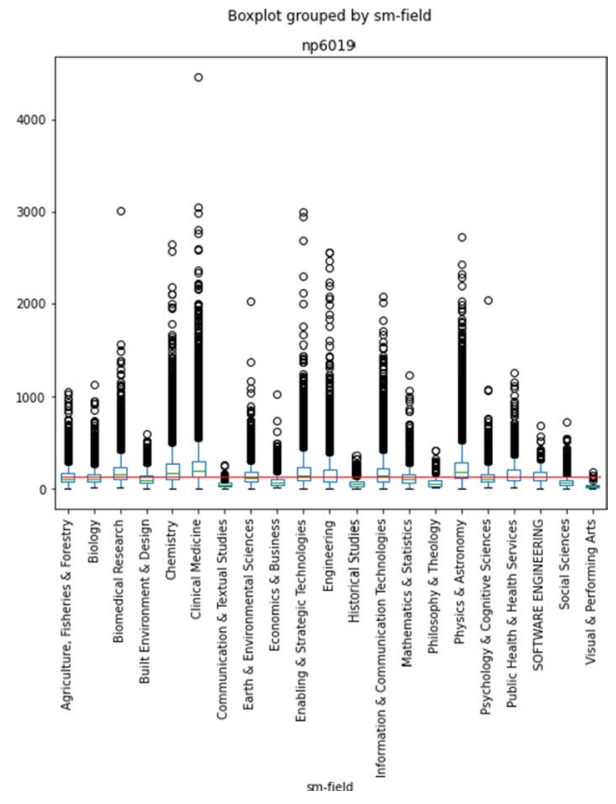

(e) np6019 - Productivity 1960-2019

Fig. 2 (continued)

Composite Citation index (c): As described earlier (see Sect. 3) the Composite Citation index is a derived measure taking several indices into consideration. Software Engineering is ranked 9th (see Table 2). Seventeen of the considered fields have higher Composite Citation index values than the top Software Engineering researcher.

Citations (np9619): Concerning total cites, Software Engineering resides on rank 10 with a median of 3600 citations. Two fields stand out with more than twice the citations of Software Engineering, namely Clinical Medicine and Biomedical Research, both with more than 7000 citations (median). When looking at outliers (Fig. 2b), we see that 16 fields have top scientists with higher cites than the most highly cited researcher in Software Engineering. Four fields stand out when looking at the outliers, namely Clinical Medicine, Biomedical Research, Engineering \& Strategic Technologies, as well as Physics \& Astronomy.

H-Index (h19): Regarding the H-Index, Software Engineering (median of 28) is also ranked in the center of the fields considered (see Fig. 2c). Here, the fields of clinical medicine and biomedical research stand out with median H-Indexes of 43 and 42, respectively. Inspecting the data distributions and outliers, we can see that 16 of the fields have a higher H-Index as the highest-rated Software Engineering researcher.

Percent of self-citations (self\%): The final measure considered is the percent of selfcitations. One should note that the authors' ranking reported the statistics with and without self-citations. As pointed out by Ioannidis et al., the ranking changed by excluding selfcitations. Authors who would have been in the top-list, considering self-citations, are not on the list with removed self-citations. Software Engineering is ranked 16th concerning the $\%$ of self-citations with a median value of $9.28 \%$. No Software Engineering Author (in Ioannidis et al. dataset) has a self-citation ratio higher than $40 \%$. On the other hand, we find several researchers with self-citations higher than 50\% in other fields. Among the 159683 top authors (all fields), 627 have more than $50 \%$ of their citations as self-citations. 


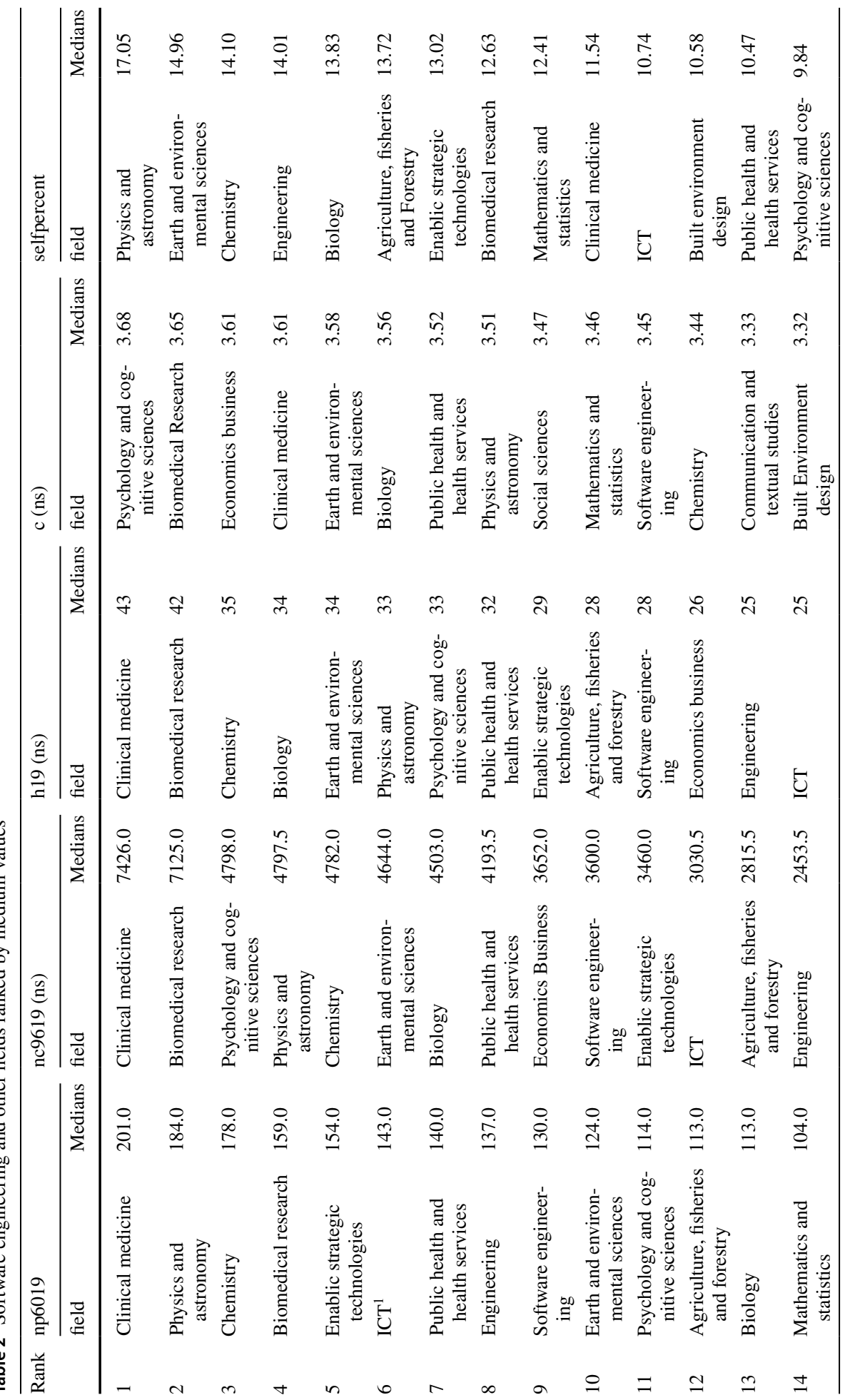




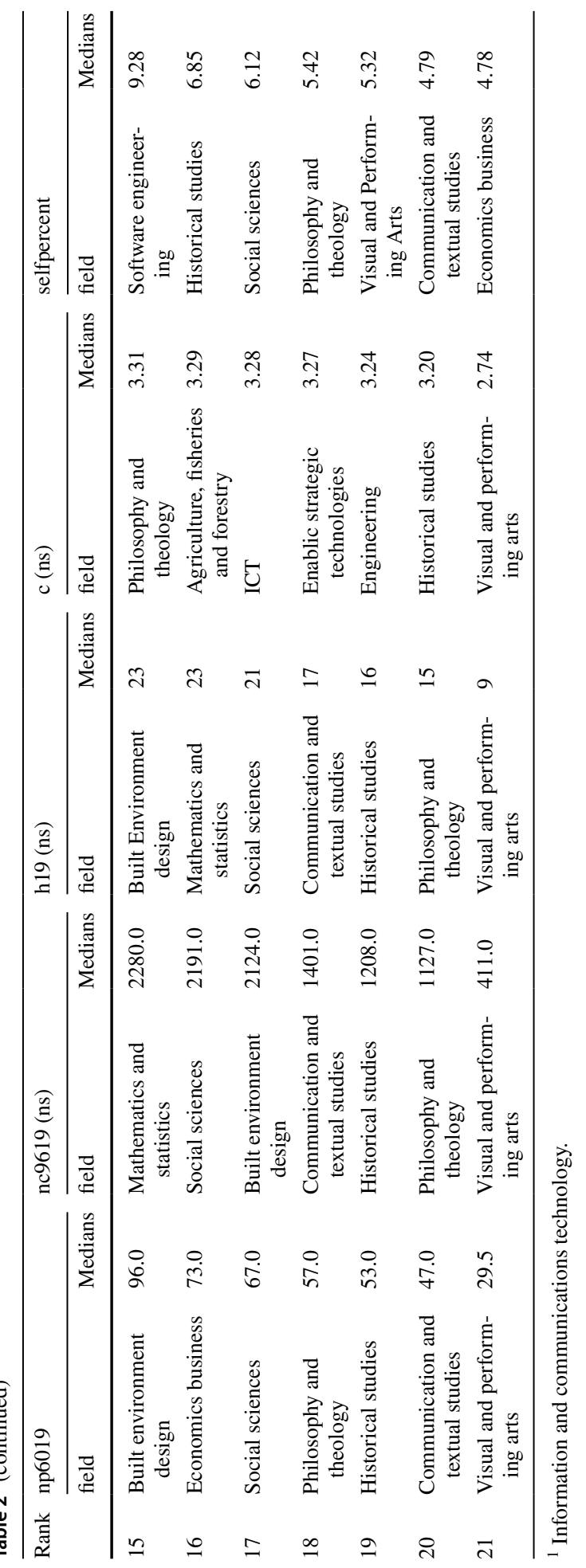


Table 3 Number of sub-fields with medians, minimum and maximum values lesser and greater than for software engineering

\begin{tabular}{|c|c|c|c|c|c|c|c|}
\hline \multirow[t]{2}{*}{ Measure ID } & \multirow[t]{2}{*}{ Measure } & \multicolumn{2}{|c|}{ Median } & \multicolumn{2}{|l|}{ Min } & \multicolumn{2}{|l|}{ Max } \\
\hline & & $<\mathrm{s}$ & $\geq S$ & $<\mathrm{S}$ & $\geq S$ & $<\mathrm{S}$ & $\geq S$ \\
\hline $\mathrm{c}(\mathrm{ns})$ & Composite cit. & 80 & 93 & 74 & 99 & 130 & 43 \\
\hline np6019 & Productivity & 89 & 84 & 30 & 143 & 64 & 109 \\
\hline nc9619 (ns) & Citations & 91 & 82 & 93 & 80 & 55 & 118 \\
\hline h19 (ns) & h-Index & 77 & 96 & 14 & 159 & 69 & 104 \\
\hline self $\backslash \%$ & Self citations & 51 & 122 & 0 & 173 & 58 & 115 \\
\hline
\end{tabular}

Productivity (np6019): The number of total papers indicates the top authors' productivity. Software Engineering is on rank 9 of 21 with median productivity of 130 papers (Table 2). Fields such as clinical medicine, physics and Astronomy, and chemistry show substantially higher values. Clinical medicine has the highest productivity with 201 papers. The data distributions visualize the outliers among the top authors with very high productivity. While the top software engineering author has less than 100 publications, a substantial number of authors in the other fields have authors with substantially more publications. For example, in the areas Clinical Medicine, Chemistry, Engineering, and Physics \& Astronomy, we find authors with more than 2000 publications.

Comparison with sub-fields: Given that each field has a number of sub-fields, we also compared software engineering with the data of the individual sub-fields. As the number of sub-fields is high, we could not show the distribution of the data side-byside. Instead, Table 3 indicates the number of sub-fields with equal or greater numbers and lesser numbers when compared with software engineering. The medians for software engineering are in the center of the median values data set except for self-citations. There, 122 sub-fields have higher medians. This was already evident when looking at the aggregated data on the field level (see Fig. 2 d. For minimum values, software engineering is also in the center of the data set for the Composite Citation index and total citations. However, for productivity and h-index, most sub-fields have higher minimumvalues. When looking at the maximum values for the Composite Citation index, the majority of sub-fields are below software engineering. However, when just looking at citations, the majority is above. For productivity, h-index, and self-citation ratio, also the majority of sub-fields are above.

Overall, the key findings of the comparison of Software Engineering with other fields can be summarized as follows:

- Within related nine sub-fields in the field Information \& Communication Technologies, Software Engineering is ranked third.

- When comparing Software Engineering with other fields, Software Engineering, lies in the center when looking at median values for the measures Total Papers (Productivity), Total Cites, H-Index, and Composite Citation index.

- Comparing the outliers (top of the top researchers) Software Engineering has considerably lower values than other fields. At least 15 or more fields have higher values than the best performing Software Engineer for the measures Total Papers (Productivity), Total Cites, H-Index, and Composite Citation index.

- In comparison to other fields, Software Engineering has a relatively low self-citation ratio of $9.28 \%$. 
Table 4 Top authors counts in software engineering per country

\begin{tabular}{lll}
\hline Group ID & Count & Countries \\
\hline 1 & 235 & USA \\
2 & 32 & Canada \\
3 & 28 & UK \\
4 & 26 & Germany \\
5 & 12 & France, Switzerland \\
6 & 11 & Italy \\
7 & 9 & China \\
8 & 8 & Australia, Israel \\
9 & 7 & Sweden \\
10 & 6 & Austria, Netherlands \\
11 & 5 & Norway \\
12 & 4 & Japan \\
13 & 3 & Korea, New Zealand, Spain \\
14 & 2 & Belgium, Ireland, Singapore \\
15 & 1 & Czechia, Finland, Greece, Hong Kong, India, \\
& & Luxembourg, Malta, Saudi Arabia, South Africa, \\
& & Taiwan \\
\hline
\end{tabular}

\section{RQ2: top-scientists}

In this section, we present the top authors in software engineering and conduct after that characterize the ranked authors analyzing the measures provided by Ioannidis et al. Specifically, we first report countries and institutions. After that, as Career Age and Total Papers are not considered in the Composite Citation index, we investigate the associations between Composite Citation index and Career Age and Total Papers, respectively.

Top authors: Table 8 shows the complete list of the top 2\% authors in Software Engineering. The total number of top authors is 441 .

Countries: Table 4 lists the number of top authors per country. The USA stands out with the number of top-ranked scientists. Canada, UK, Germany, France, Switzerland, and Italy have more than ten scientists on the top list. It is also noteworthy that smaller countries (population with less than 10 million persons) are present who have multiple top authors, such as Switzerland (8.5 million), Israel (9.1 million), Sweden (10.2 million), Austria (8.9 million), and Norway (5.3 million).

Institutions: Table 5 shows the number of top scholars per institution. The last known institution/affiliation was used. The majority of institutions listed (172) only have one scholar working with them. We see that both universities and companies have active and hence recognized researchers in the top author list. The company with the most scholars is Microsoft Research (15 scholars), followed by Google LLC (9 scholars). Other companies highlighted are NVIDIA (7 scholars), Adobe Inc. (4 scholars), Pixar Animation Studios (4 scholars), and Facebook Inc. (2 scholars). The universities with the most scholars are the University of Washington, Carnegie Mellon University, Georgia Institute of Technology, with more than seven scholars each, and Stanford University, The University of British Columbia, and University of California, Davis. 


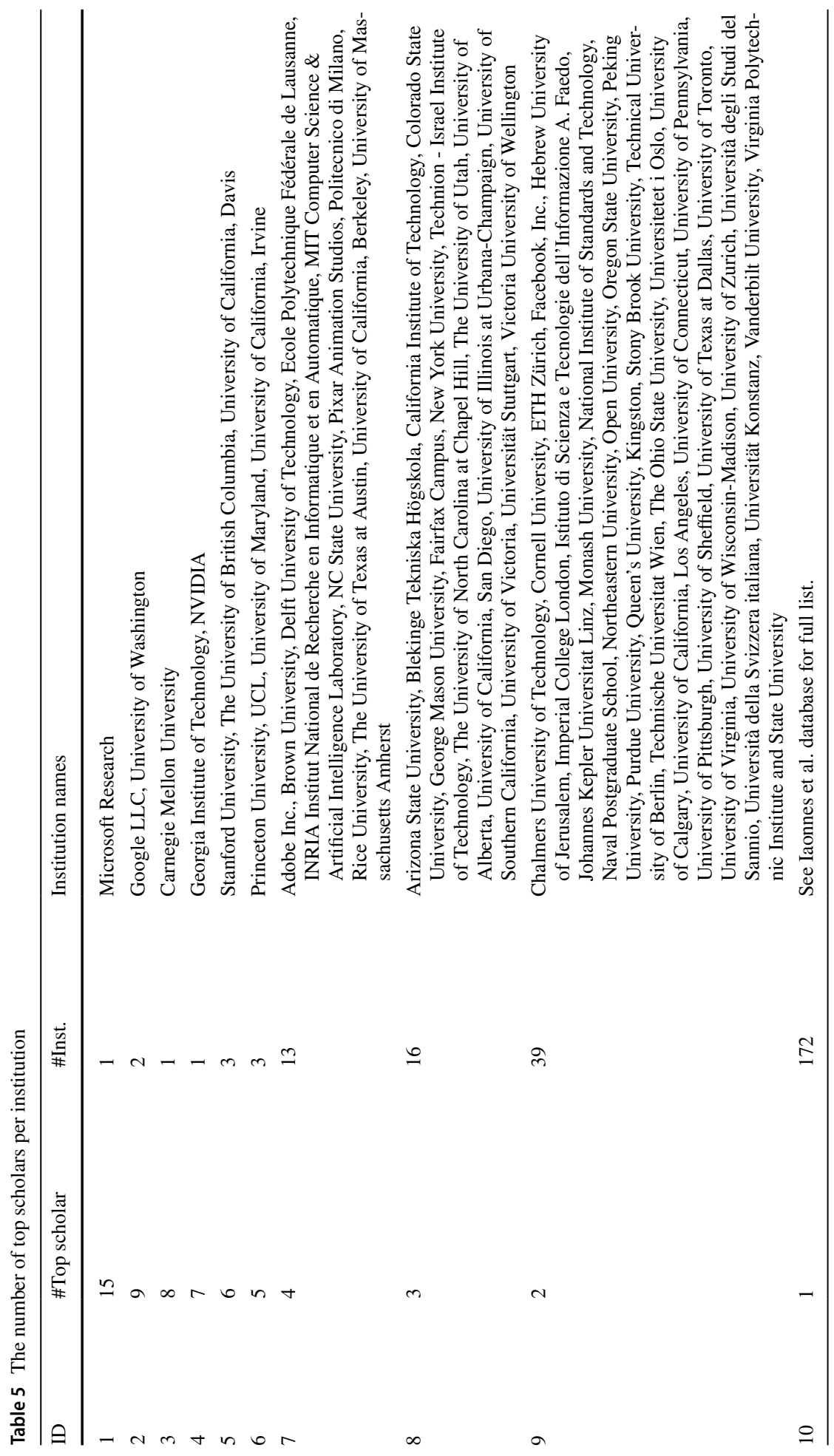


Fig. 3 Total papers (np6019) versus composite citation index

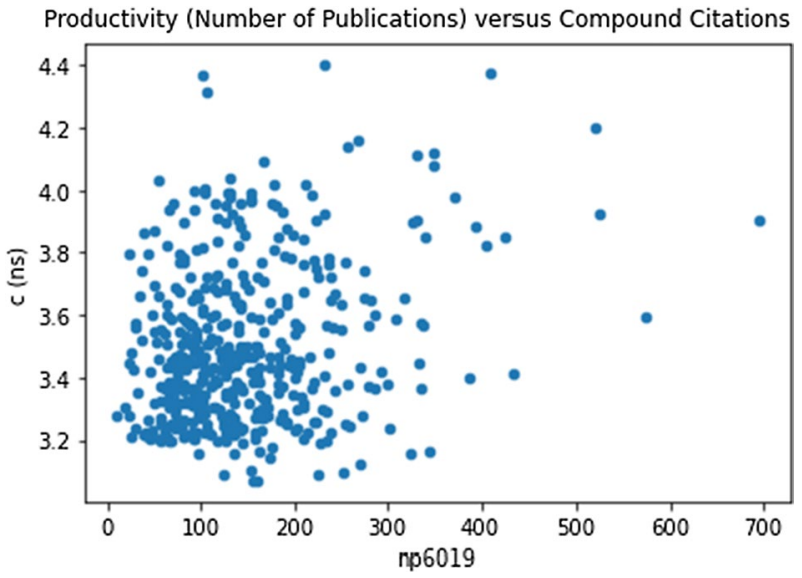

Fig. 4 Distribution of career age

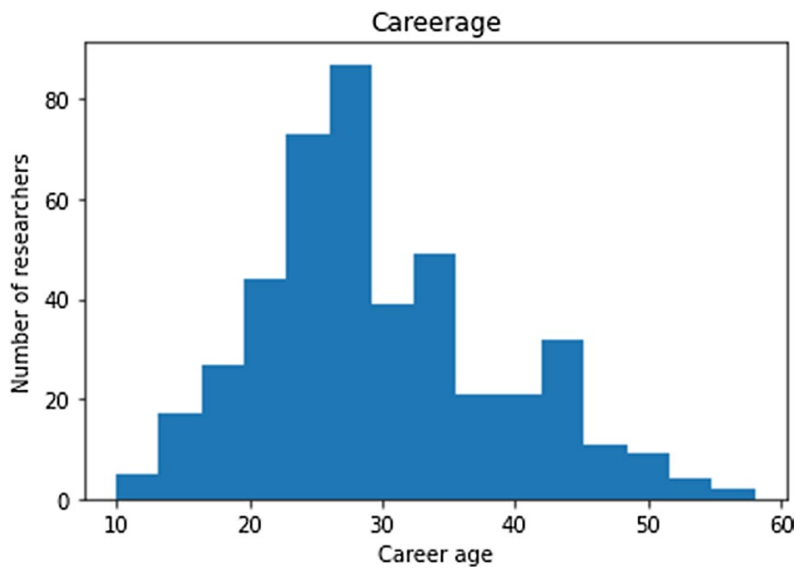

Productivity and Composite Citation index: Figure 3 shows a scatter plot of the total papers produced in the time period 1960-2019 (np6019) and Composite Citation index $(\mathrm{c}(\mathrm{ns}))$. Visually the figure shows that there is no or little apparent association between the total number of papers produced and the Composite Citation index. Calculating the correlation between the two variables, we also see a very weak positive correlation (Kendall's $\tau=0.091$ ).

Career age and composite citation index: Figure 4 shows the age profile of the career age of the top software engineering researchers. The figure shows that the majority of researchers have a career age of 25-35. However, when looking at the association between career age and Composite Citation index, we observe a similar pattern for productivity (papers published), i.e., the data lack association. This lack of association is also evident from the weak correlation (Kendall's Tau $=0.102$ ). However, this may also be an artefact, given that the age of publications is not accounted for in the analysis (Fig. 5). 
Fig. 5 Career age versus composite citation index

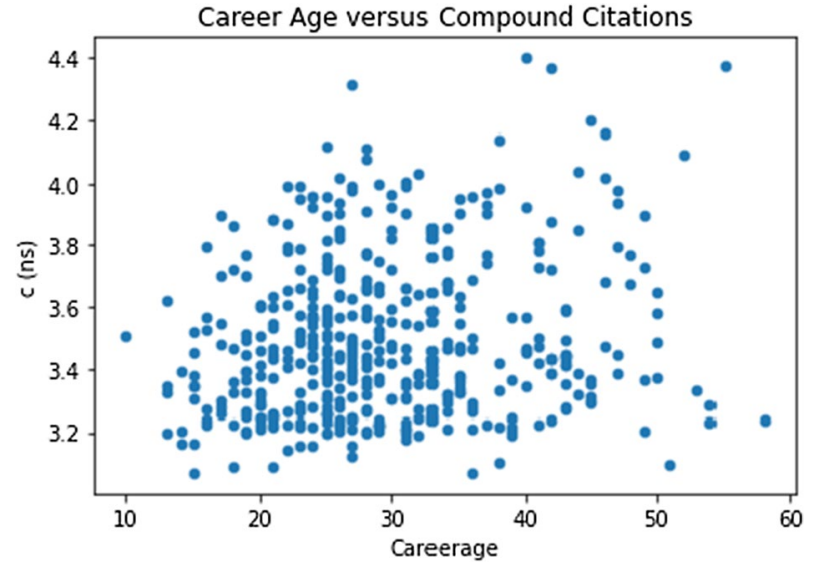

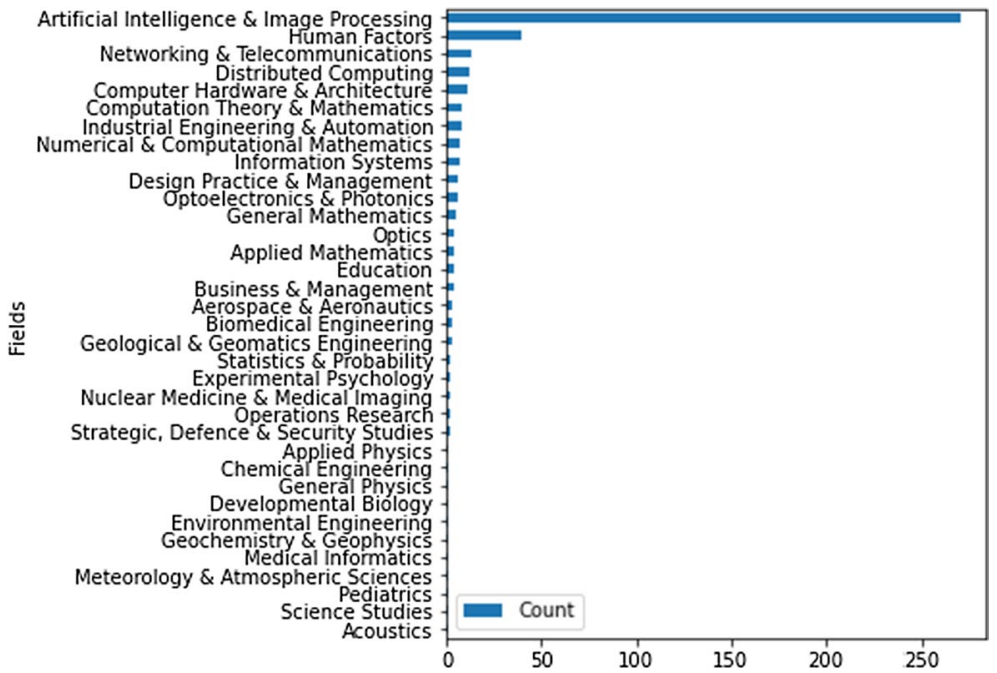

Fig. 6 Frequency of related fields occurring for top-authors

\section{RQ3: Activity of top authors in other fields}

As described earlier (see Table 1), in the data provided by Ioannidis et al., the author profiles were assigned two fields, one being the primary and the other the secondary sub-field. Figure 6 shows the count of secondary sub-fields for software engineering researchers.

Artificial Intelligence \& Image Processing stands out as the secondary sub-field occurring most of the time. The second most frequently identified sub-field is Human Factors. After that, several sub-fields related to other computing areas (such as Distributed Computing or Computer Hardware \& Architecture) are next based on counts. Fields further away from software engineering (e.g., General Physics, Development Biology, or Acoustics) are only occurring once.

Concerning the top authors as identified by Ioannidis et al., we highlight the following findings: 
Table 6 Assessment of accurate classification of researchers in the top-list

\begin{tabular}{lll}
\hline & Total & Percent \\
\hline In SE & 278 & 63 \\
Not in SE & 163 & 37 \\
& 441 & 100 \\
\hline
\end{tabular}

- Overall, 411 top authors were identified. The top three authors are Terzopoulos, Demetri, Boehm, Barry, and Levoy, Marc.

- The top three countries with the most top authors are the USA, Canada, and the UK.

- Concerning institutions, companies as well as universities are at the top. Microsoft leads with the highest number of top scholars (15), followed by Google LLC and the University of Washington.

- Productivity and career age are not associated with Composite Citation index, as indicated by correlation analysis.

- Artificial Intelligence \& Image Processing is the most strongly associated second field for researchers with software engineering as their main field.

\section{Discussion}

When presenting the results, we kept the data as is, without filtering or manipulating the data. The reason was not to introduce biases in the analysis. After that, we reviewed the data set's details to reflect on the set for our particular case, i.e., software engineering.

That is, in the discussion, we focus on two aspects when analyzing the results. First, we reviewed the top researchers' list to identify whether all are indeed in software engineering, given that the fields and sub-fields have been assigned using a trained machine-learning model. Second, we compared our findings with the rankings presented in the related work.

\section{Reflections on the top-list}

Looking at the authors, we classified them to either be in software engineering or not software engineering using Google Scholar profiles. If no profile was available, we identified the researchers' webpages. We looked at the research topics specified by the authors and their publication lists. Table 6 shows the analysis outcome. The focus was on identifying the researchers' main area, given that the main sub-field was under consideration. If software engineering is not the researchers' main area, this does not mean that the researchers classified with "Not in SE" have not done any software engineering research.

Thus, this is an indication that the machine-learning classifier was not perfect. In this step, we only detected that people with software engineering not being their main field appeared in the software engineering list.

For example, when looking at the top-list (see Table 8 in the Appendix), we see that among the top researchers, we have three cases of authors (Terzopoulos, Demetri; Levoy, Marc; Hoppe, Hugues) working in computer graphics. They all were classified in their second field as working with Artificial Intelligence \& Image Processing. Thus, it appears that in some cases, the priorities in the fields (main field and second field) were not correct. 


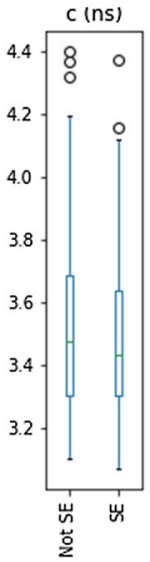

(a) c

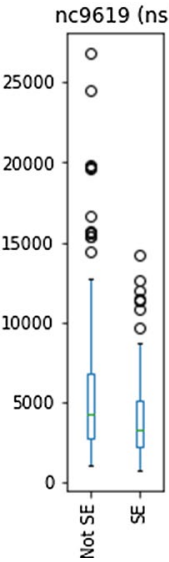

(b) nc9619

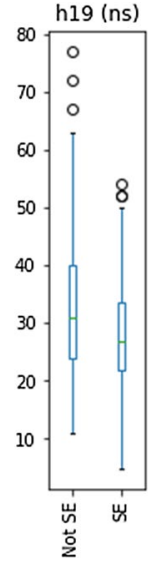

(c) h19

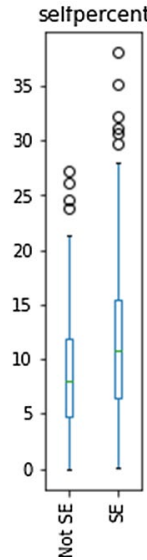

(d) Self\%

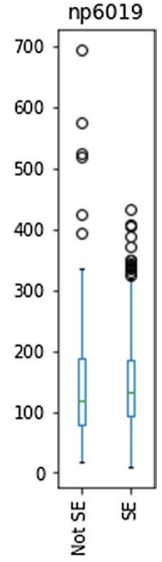

(e) $\mathrm{np} 6019$

Fig. 7 Comparison of true positives (software engineering researchers) and false positives (non-software engineering researchers) in the software engineering data set

When one analyzes the top researchers in computer graphics, these researchers would be missing in that analysis when looking at the main field.

However, it is essential to point out that many researchers were to be analyzed, requiring an automatic classification approach. Reviewing the data, we suspect that the classifier may have associated graphics and visualization in general with the strong focus of software engineering on end-user experience and usability and software visualization (e.g., researcher Eick, Stephen G.) as almost all researchers classified wrongly were associated with image processing, computer graphics, or visualization. Note that this is an interpretation observing the fields, though, given the nature of the model (convolutional neural networks) used for classification, it is retrospectively impossible to explain why the classifier produced the false positives.

Next, we analyze the impact on our analysis by comparing the SE-researchers with the non-SE researchers. In particular, we briefly describe how software engineering data was impacted by the inclusion of false positives (i.e., researchers classified as software engineers but should not have been). Figure 7 shows that the false positives impacted the software engineering data set. In particular, for Composite Citation index (c), total cites (nc9619), and h-index (h19), the values are moderately higher compared to the true positives. We also see that the extreme values (outliers) are higher for c, nc6919, and h19. For self-citations and total papers, the median values are slightly higher for the true positives.

When looking at the impact of the new median values on the ranking compared to other fields, the ranking in Table 2 would change. For Composite Citation index (new median = 3.432 ) and h-Index (new median $=27$ ), the rank would be reduced by one. For Total Citations (np6919, new median $=3302$ ), the rank would be reduced by two. Concerning Total Papers (np6019, new median $=133$ ), there is no change in rank. However, for Percent of Self-Citations, the largest change occurs, increasing software engineering's rank by five (new median $=10.75 \%$ ). In Table 8 in Appendix A we highlighted the correctly classified software engineering researchers using $(*)$. 
Next, we compare the ranking based on the database provided by Ioannidis et al. with the already published rankings of software engineering scholars.

\section{Comparison with related work}

Two prominent rankings focused on identifying the top scholars in software engineering. The first is the JSS-Ranking, which was published for different time intervals (see Sect. 2). Furthermore, Fernandes (2014) published a ranking covering the years 1971-2012.

JSS-rankings: The first JSS-ranking has been published in 1994 (Glass 1994). Thereafter, each year the ranking was extended by 1 year (e.g., 1993-1994 Glass 1995 and thereafter 1993-1995 Glass 1996). Later, not only the last year was incremented, but also the first year (see e.g. rankings 1993-1997 Glass 1998 and 1994-1998 Glass 1999. The last ranking using consistent measures and schedules was published in 2011 (Wong et al. 2011). A new ranking was published after a break, using new scientific measures (Karanatsiou et al. 2019). To compare the findings, we first compare the series from 1993 to 2008 with our findings and compare with the latest ranking (cf. Karanatsiou et al. 2019).

Regarding the rankings from 1993 to 2008, the rankings were only based on the journals that were considered the top ones in software engineering. Each paper published by an author was counted differently depending on the number of co-authors. A single author would receive one point for a paper. For co-authors, first fractional contributions were calculated (e.g., having three authors would give 0.33 points for each author). The fractional values were transformed (e.g., a fractional contribution of 0.33 would become 0.5 ) to not penalize co-authorship. Given that the ranking is purely focused on productivity (number of papers published), we should not expect that most JSS ranking authors appear in the database by Ioannidis, where the focus was on a Composite Citation index (c). However, we expect some overlap and may see whether researchers appearing in the list are in the list by Ioannidis et al., but not classified as software engineers.

Table 7 provides an overview of the ranking results, focusing on the rankings for the time periods 1993-1997 (Glass 1998) until the last ranking (Wong et al. 2011), which included the time periods 2003-2007 as well as 2004-2008. The table shows the ranks the researchers achieved in a particular ranking. In case an author did not appear in the ranking, this is indicated using the bullet-symbol $(\bullet)$. In the outer right column, we state whether the authors in the JSS ranking appeared in the ranking by Ioannidis et al. If a rank-number is stated, they appeared in the software engineers' list, whose fields were correctly classified as software engineering. In case the author did not appear, we used the bullet-symbol again $(\bullet)$.

We also show which authors were found in the top-list we generated based on the database by Ioannidis et al.

We distinguish between finding the authors among those classified as software engineering researchers in their main field (the rank number is stated), or researchers not classified as software engineers in their main field, but included and not classified as software engineers in the database $(\sqrt{ })$. As is shown in Table 721 of the $54(39 \%)$ researchers that were classified by Ioannidis et al. as being in software engineering (primary field)) were also ranked by JSS. We also found six researchers (11\%) in the JSS ranking but not classified with software engineering as their primary field in the database. 


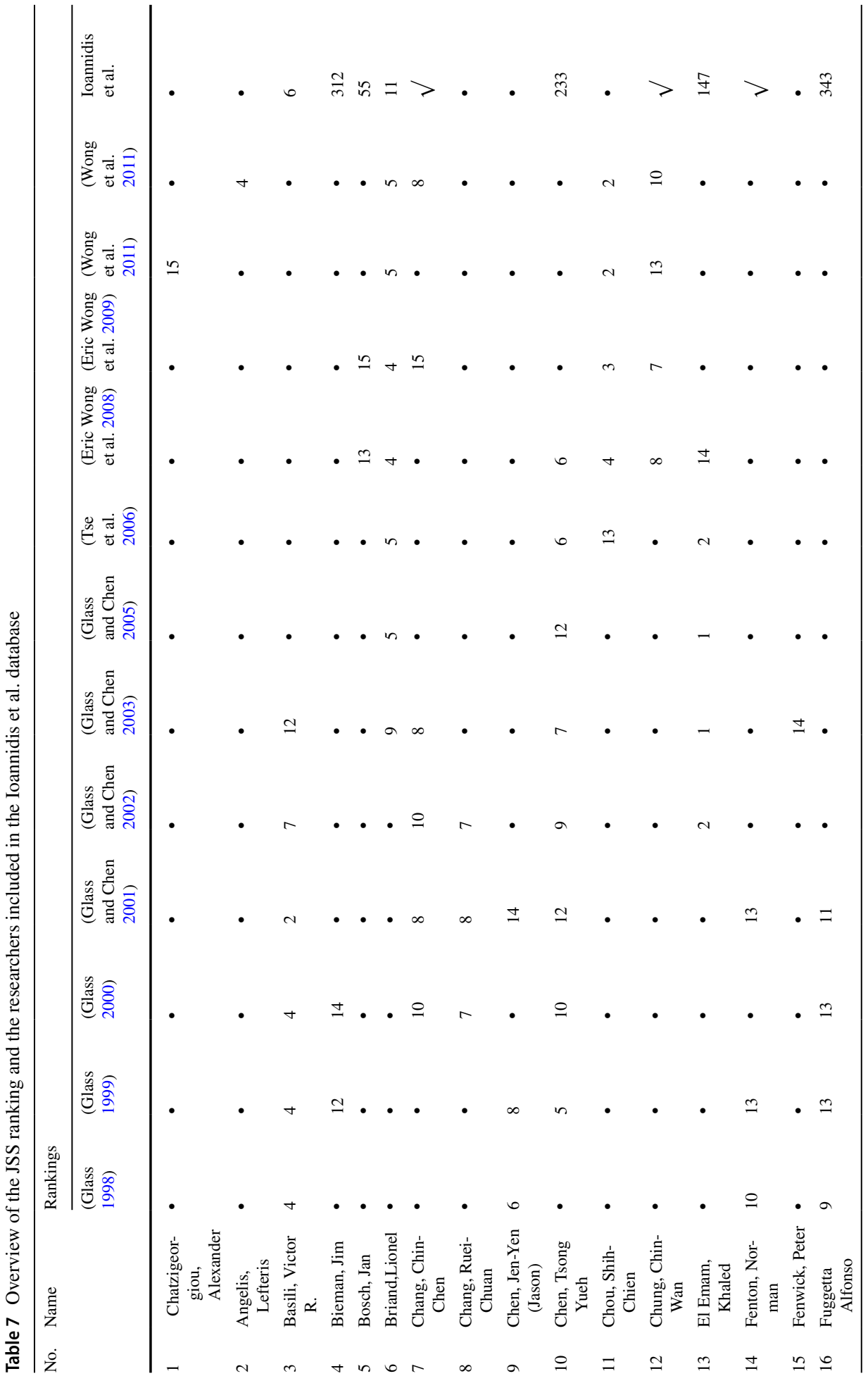




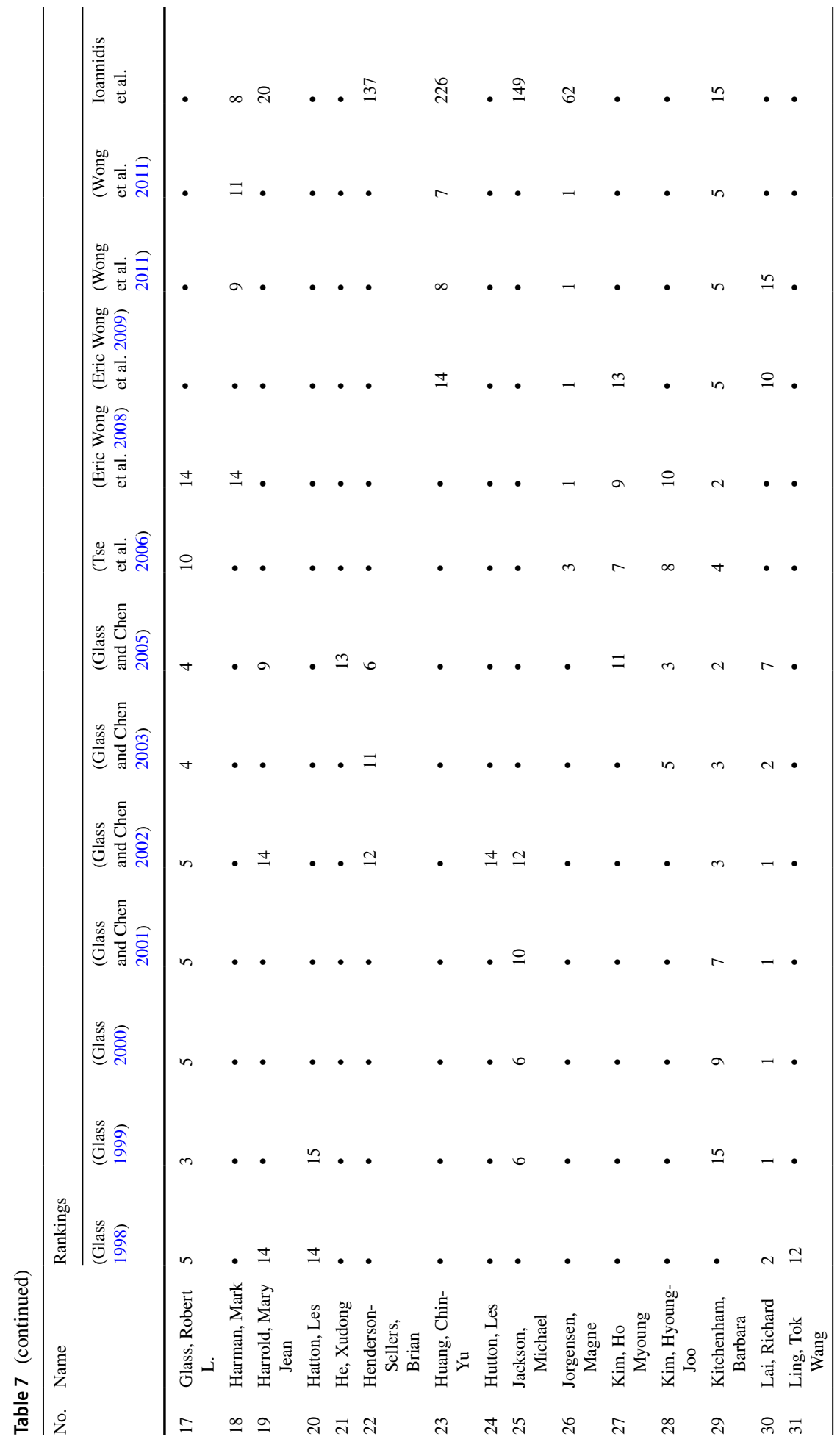




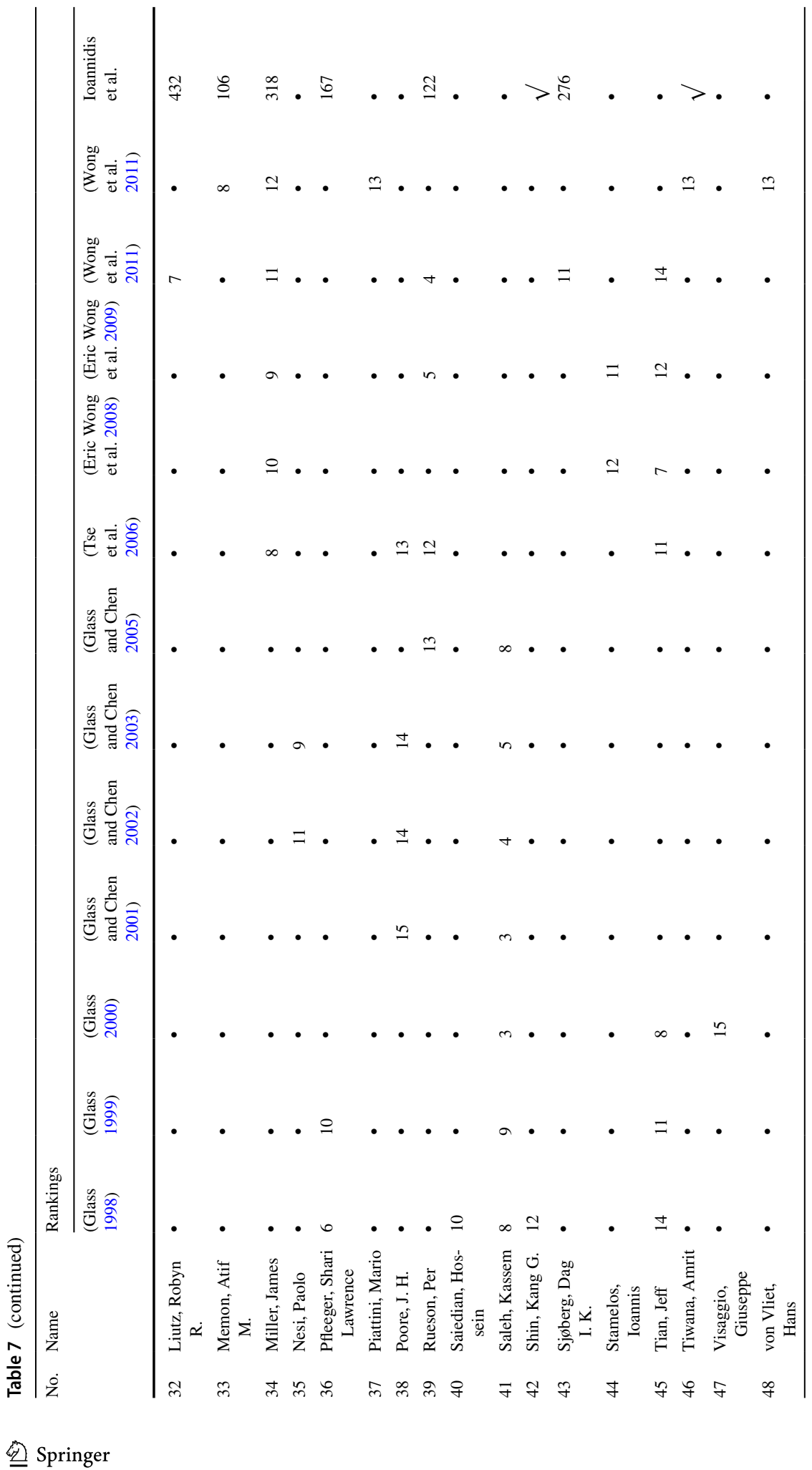




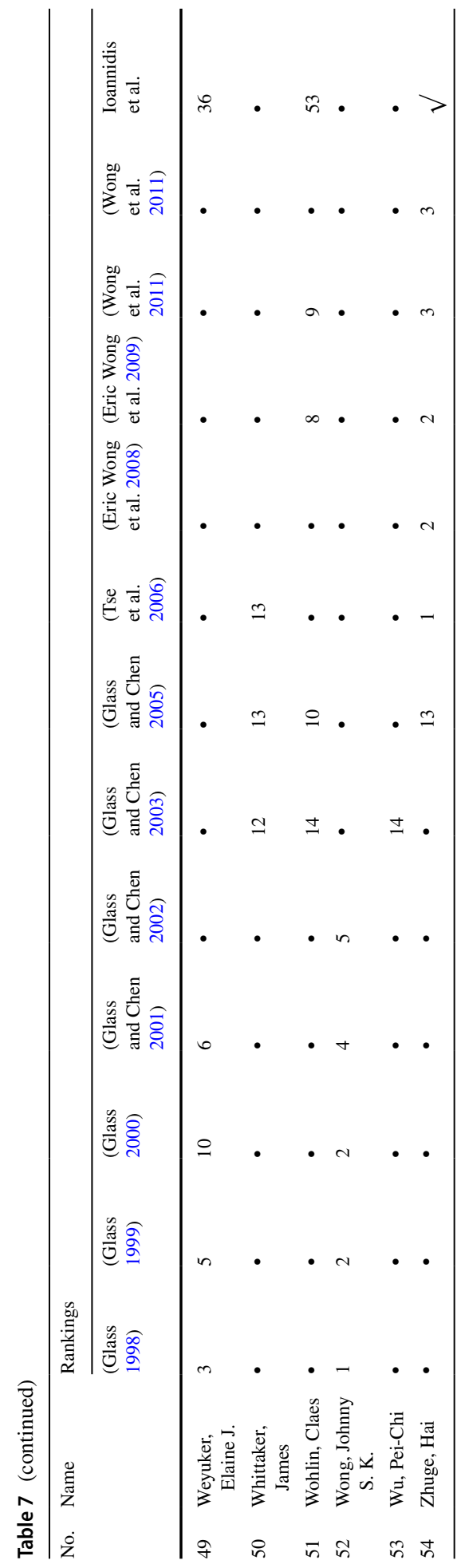


Next, we compare the most recent JSS (Karanatsiou et al. 2019) ranking with Ioannidis et al.'s database. The newest JSS ranking is analyzed separately as the methodology for ranking the scientists has changed. Key changes are:

- The authors did not focus on the predefined set of journals from the earlier rankings but substantially extended the set of journals considered. They also included conferences.

- Researcher productivity was now measured as total paper count.

- Researchers were assigned to three groups based on their career age (experienced researchers with more than 12 years of research, consolidated researchers with 8-12 years of research, and early-stage researchers with up to 7 years of research)

- New analyses were included by considering several types of rankings based on (a) top journal publications, (b) top publications (including journals and conferences), and (c) impact measured in citations.

For the comparison with the database, we only considered the list of experienced researchers, given that the database covered the period of 1960-2019 and mainly included researchers in the experienced category (see Fig. 4). Overall, 45 researchers classified as experienced were listed in the last JSS ranking. Of these 45 researchers, 28 (62\%) were included in the Ioannidis et al. database, all classified correctly as software engineering researchers.

Ranking by Fernandes (2014): The ranking by Fernandes assessed researchers by productivity. The data was obtained by focusing on the DBLP database (Ley 2009). Fernandes identified 30 top researchers in the period 1971-2012. Given that a more extended period is covered, this ranking is a particularly interesting reference for comparison. Of the 30 researchers, 24 were included in the database and classified as software engineering researchers. One researcher ranked by Fernandes (Taghi M. Khoshgoftaar) was found in the Ioannidis et al. database but not classified as software engineering in his main field. Overall, $83 \%$ of the researchers in the ranking by Fernandes are also included in the database.

Fernandes used fractional and harmonic citations (Hagen 2014), which consider relative author contributions. However, no impact (citations) was measured. In the Composite Citation index used by Ioannidis, also author contributions are distinguished. It is noteworthy that one measure (ncsf: total citations to papers for which the researcher is the sole or the first author) used would not apply to software engineering. In software engineering, the last author position would usually be considered the least contributing, while in the medical field, the last author position stands out (Baerlocher et al. 2007).

Overall, the analysis of the database for software engineering shows that when using the database in another field, it may be necessary to investigate researchers' classification. Researchers wrongly classified as being in the investigated field may be easier to detect than researchers not appearing in the field due to wrong classifications. We used existing rankings to detect researchers not appearing in software engineering. However, previous rankings may not be available in other fields, which would make the detection of missing researchers more difficult. 


\section{Conclusion}

We analyzed the top-ranked software engineering researchers in the database published by Ioannidis et al. The database provided the opportunity to gain novel insights on the top authors in software engineering, as a new measure was used to identify the authors (Composite Citation index), and that the ranking was considering the number of self-citations. Furthermore, the database included other fields that were assessed using the same measures. Therefore, software engineering researchers could learn how they compare to other fields.

We formulated three research questions, which are briefly answered as follows:

RQ1: How do author citations in software engineering compare to other fields concerning the citation measures provided by Ioannidis et al.? We first compared software engineering with nine other related fields in the area of Information \& Communication Technology, where it was ranked third concerning Compound Citations. After that, we compared software engineering with 20 other fields included in the database. Software engineering lies in the center of the fields for Total Papers, Total Cites, H-Index, and Composite Citation index. Given that $37 \%$ of the researchers were wrongly classified as software engineers in their main field, there is an effect on the comparison. The effect was minor for most measures, except the percentage of self-citations.

What are the top authors' demographics (e.g., country of origin, institutions, etc.)? In total, Ioannidis et al. identified 441 researchers as top researchers in software engineering. However, after examining the authors' list in further detail, only 278 authors were in software engineering. By looking at other rankings done in software engineering, we identified seven additional top researchers that were not classified as software engineers in their main field. The top researcher in the field of software engineering is Barry Boehm. In our reporting, we also highlighted those researchers in the ranking that are software engineers. The top countries and institutions were also identified. The top countries were the USA, Canada, and the UK. The institution with most software engineering top authors was Microsoft. We also found that career age and productivity were not associated with the Composite Citation index, which may be also attributed to not accounting for publication age, which was not included in the ranking.

In which other fields software engineering authors are active? In total, 33 fields were listed as second fields for researchers classified as software engineers in their main field. The most frequent field that occurred together with software engineering was Artificial Intelligence \& Image Processing, followed by Human Factors.

Overall, given that a large portion of researchers classified as software engineers were not in software engineering, we suggest that other fields using the database for their rankings need to review the author lists. As the classification was done using convolutional neural networks, there may always be a portion of not correctly classified researchers. However, overall, $60 \%-80 \%$ of the researchers listed in other software engineering rankings were identified, there is some confidence in the identification of relevant researchers.

In future work, we suggest using the database further to assess researchers in other fields, e.g., taking into account the conventions in the field regarding the author order as weighted in the Composite Citation index and the reliability of underlying classification of the researchers. 
Table 8 Top author list software engineering

\begin{tabular}{|c|c|}
\hline Rank & Author \\
\hline 1 & Terzopoulos, Demetri \\
\hline 2 & Boehm, Barry* \\
\hline 3 & Levoy, Marc \\
\hline 4 & Hoppe, Hugues \\
\hline 5 & Guibas, Leonidas \\
\hline 6 & Basili, Victor* \\
\hline 7 & Slater, Mel \\
\hline 8 & Harman, Mark* \\
\hline 9 & Keim, Daniel \\
\hline 10 & Parnas, David Lorge* \\
\hline 11 & Briand, Lionel* ${ }^{*}$ \\
\hline 12 & Wadler, Philip* \\
\hline 13 & Witkin, Andrew \\
\hline 14 & Kobbelt, Leif \\
\hline 15 & Kitchenham, Barbara* \\
\hline 16 & Turk, Greg \\
\hline 17 & Godefroid, Patrice* \\
\hline 18 & Hertzmann, Aaron \\
\hline 19 & Fedkiw, Ronald \\
\hline 20 & Harrold, Mary Jean* \\
\hline 21 & Taubin, Gabriel \\
\hline 22 & Hanrahan, Pat \\
\hline 23 & Funkhouser, Thomas \\
\hline 24 & Schmidt, Douglas C.* \\
\hline 25 & Ware, Colin \\
\hline 26 & Gleicher, Michael* \\
\hline 27 & Nielsen, Jakob* \\
\hline 28 & Zeller, Andreas* \\
\hline 29 & Lam, Monica S.* \\
\hline 30 & Rusinkiewicz, Szymon \\
\hline 31 & Herbsleb, Jim* \\
\hline 32 & Debevec, Paul \\
\hline 33 & Van Lamsweerde, Axel* \\
\hline 34 & Holzmann, Gerard J.* \\
\hline 35 & Farouki, Rida T. \\
\hline 36 & Weyuker, Elaine J.* \\
\hline 37 & Manocha, Dinesh \\
\hline 38 & Durand, Frédo \\
\hline 39 & Ball, Thomas* \\
\hline 40 & Van Wijk, Jarke \\
\hline 41 & Hassan, Ahmed E.* \\
\hline 42 & Garlan, David* \\
\hline 43 & Seidel, Hans Peter \\
\hline 44 & Brooks, Frederick P.* \\
\hline 45 & Cohen-Or, Daniel \\
\hline 46 & Sen, Koushik* \\
\hline
\end{tabular}


Table 8 (continued)

\begin{tabular}{|c|c|}
\hline Rank & Author \\
\hline 47 & Raskar, Ramesh \\
\hline 48 & Alexa, Marc \\
\hline 49 & Taylor, Richard* \\
\hline 50 & Shewchuk, Jonathan Richard \\
\hline 51 & Fattal, R. \\
\hline 52 & Cohen, Michael F. \\
\hline 53 & Wohlin, Claes* \\
\hline 54 & Stasko, John \\
\hline 55 & Bosch, Jan* \\
\hline 56 & Gross, Markus \\
\hline 57 & Hodgins, Jessica \\
\hline 58 & Flanagan, Cormac* \\
\hline 59 & Ma, Kwan Liu* \\
\hline 60 & Kemerer, Chris F.* \\
\hline 61 & Fitzgerald, Brian* \\
\hline 62 & Jørgensen, Magne* \\
\hline 63 & Sederberg, Thomas W. \\
\hline 64 & Batory, Don* \\
\hline 65 & Pugh, William* \\
\hline 66 & Azuma, Ron \\
\hline 67 & Reynolds, John C.* \\
\hline 68 & Ramamoorthi, Ravi \\
\hline 69 & Necula, George* \\
\hline 70 & Meyer, Bertrand* \\
\hline 71 & Sutcliffe, Alistair* \\
\hline 72 & Nuseibeh, Bashar* \\
\hline 73 & Pottmann, Helmut \\
\hline 74 & Thomas, D. A. \\
\hline 75 & Dybå, Tore* \\
\hline 76 & Leroy, Xavier* \\
\hline 77 & Larus, James* \\
\hline 78 & Tonella, Paolo* \\
\hline 79 & Aiken, Alex* \\
\hline 80 & Rinard, Martin* \\
\hline 81 & Stam, Jos \\
\hline 82 & Bajaj, Chandrajit \\
\hline 83 & Kruchten, Philippe* \\
\hline 84 & Levin, David \\
\hline 85 & Kramer, Jeffrey* \\
\hline 86 & Zimmermann, Thomas* \\
\hline 87 & Mockus, Audris* \\
\hline 88 & Myers, Andrew C.* \\
\hline 89 & Garland, Michael* \\
\hline 90 & Williams, Laurie* \\
\hline 91 & Mens, Tom* \\
\hline
\end{tabular}


Table 8 (continued)

\begin{tabular}{|c|c|}
\hline Rank & Author \\
\hline 92 & Gulwani, Sumit* \\
\hline 93 & Heer, Jeffrey* \\
\hline 94 & Wilkinson, Leland \\
\hline 95 & Jackson, Daniel* \\
\hline 96 & Korel, Bogdan* \\
\hline 97 & Shamir, Ariel \\
\hline 98 & Rossignac, Jarek \\
\hline 99 & Shaw, Mary* \\
\hline 100 & Holt, R. C.* \\
\hline 101 & Appel, Andrew W.* \\
\hline 102 & Perlin, Ken* \\
\hline 103 & Shepperd, Martin* \\
\hline 104 & Spinellis, Diomidis* \\
\hline 105 & Kass, Michael \\
\hline 106 & Memon, Atif* \\
\hline 107 & Barr, Alan H. \\
\hline 108 & Blinn, James F. \\
\hline 109 & Robillard, Martin P.* \\
\hline 110 & Czarnecki, Krzysztof* \\
\hline 111 & Gupta, Rajiv* \\
\hline 112 & De Lucia, Andrea* \\
\hline 113 & Curless, Brian* \\
\hline 114 & Kaufman, Arie \\
\hline 115 & Johnson, Ralph \\
\hline 116 & Storey, Margaret Anne* \\
\hline 117 & Binkley, David \\
\hline 118 & Lehman, M. M.* \\
\hline 119 & Medvidovic, Nenad* \\
\hline 120 & Owens, John D. \\
\hline 121 & Menzies, Tim* \\
\hline 122 & Runeson, Per* \\
\hline 123 & Arcuri, Andrea* \\
\hline 124 & Devanbu, Premkumar* \\
\hline 125 & Lévy, Bruno \\
\hline 126 & Lanza, Michele* \\
\hline 127 & Xie, Таo* \\
\hline 128 & Sheffer, Alla \\
\hline 129 & Wattenberg, Martin* \\
\hline 130 & Ward, Gregory J. \\
\hline 131 & Seli_, Bran* \\
\hline 132 & Murphy, Gail C.* \\
\hline 133 & Munzner, Tamara \\
\hline 134 & Thalmann, Daniel \\
\hline 135 & Kiczales, Gregor* \\
\hline 136 & Felleisen, Matthias* \\
\hline
\end{tabular}


Table 8 (continued)

\begin{tabular}{|c|c|}
\hline Rank & Author \\
\hline 137 & Henderson-Sellers, Brian* \\
\hline 138 & Pai, Dinesh K. \\
\hline 139 & Aho, Alfred* \\
\hline 140 & Igarashi, Takeo \\
\hline 141 & Egyed, Alexander \\
\hline 142 & Purchase, Helen \\
\hline 143 & Fekete, Jean Daniel* \\
\hline 144 & Baraff, David \\
\hline 145 & Zorin, Denis \\
\hline 146 & McMinn, Phil* \\
\hline 147 & El Emam, Khaled* \\
\hline 148 & Pfister, Hanspeter \\
\hline 149 & Tsai, Wei Tek* \\
\hline 150 & $\mathrm{Hu}$, Shi Min \\
\hline 151 & Reiss, Steven* \\
\hline 152 & Carpendale, Sheelagh* \\
\hline 153 & Heckbert, Paul S.* \\
\hline 154 & Hierons, Robert M.* \\
\hline 155 & Schröder, Peter \\
\hline 156 & Canfora, Gerardo* \\
\hline 157 & Horwitz, Susan* \\
\hline 158 & Gotsman, Craig J. \\
\hline 159 & Farin, Gerald \\
\hline 160 & Müller, Matthias* \\
\hline 161 & Harris, Tim* \\
\hline 162 & Ernst, Michael D.* \\
\hline 163 & Elber, Gershon \\
\hline 164 & Lindstrom, Peter \\
\hline 165 & Eppler, Martin J. \\
\hline 166 & Nagappan, Nachiappan* \\
\hline 167 & Pfleeger, Shari Lawrence* \\
\hline 168 & Easterbrook, Steve* \\
\hline 169 & Offutt, A. J. \\
\hline 170 & Jensen, Henrik Wann \\
\hline 171 & Offutt, Jeff** \\
\hline 172 & Agarwala, Aseem \\
\hline 173 & Chambers, Craig \\
\hline 174 & Amenta, Nina \\
\hline 175 & Weimer, Westley* \\
\hline 176 & Sorkine, Olga* \\
\hline 177 & Dodgson, Neil A. \\
\hline 178 & Luebke, David \\
\hline 179 & Sommerville, Ian* \\
\hline 180 & Desbrun, Mathieu \\
\hline 181 & Finkelstein, Anthony* \\
\hline
\end{tabular}


Table 8 (continued)

\begin{tabular}{|c|c|}
\hline Rank & Author \\
\hline 182 & Yu, Yizhou \\
\hline 183 & Ebert, Christof* \\
\hline 184 & Westermann, Rüdiger \\
\hline 185 & Cleland-Huang, Jane* \\
\hline 186 & Littlewood, Bev* \\
\hline 187 & Koschke, Rainer* \\
\hline 188 & Hughes, John* \\
\hline 189 & Erwig, Martin* \\
\hline 190 & Popovi_,Zoran \\
\hline 191 & Paris, Sylvain \\
\hline 192 & Antoniol, Giuliano* \\
\hline 193 & Johnson, Chris* \\
\hline 194 & Kajiya, Jim \\
\hline 195 & North, Chris* \\
\hline 196 & Pinkall, Ulrich \\
\hline 197 & Tichy, Walter F.* \\
\hline 198 & Ryder, Barbara G.* \\
\hline 199 & McKinley, Kathryn S.* \\
\hline 200 & $\mathrm{Ju}, \mathrm{Tao}$ \\
\hline 201 & Anton, Annie* \\
\hline 202 & Wong, Kenny* \\
\hline 203 & Visser, Eelco* \\
\hline 204 & Abrahamsson, Pekka* \\
\hline 205 & Warren, Joe \\
\hline 206 & Hart, J. \\
\hline 207 & Kuhn, D. Richard* \\
\hline 208 & Bertolino, Antonia* \\
\hline 209 & Zyda, Michael \\
\hline 210 & Maiden, Neil* \\
\hline 211 & Krinke, Jens* \\
\hline 212 & Bridson, Robert \\
\hline 213 & Badler, Norman I. \\
\hline 214 & Rosenblum, David S.* \\
\hline 215 & Scacchi, Walt* \\
\hline 216 & Seaman, Carolyn* \\
\hline 217 & Curtis, Bill* \\
\hline 218 & van Deursen, Arie* \\
\hline 219 & Lipman, Yaron \\
\hline 220 & Schmalstieg, Dieter \\
\hline 221 & Zave, Pamela* \\
\hline 222 & Nielson, Gregory M. \\
\hline 223 & Max, Nelson \\
\hline 224 & Pajarola, Renato \\
\hline 225 & Foxlin, Eric \\
\hline 226 & Huang, Chin $\mathrm{Yu}^{*}$ \\
\hline
\end{tabular}


Table 8 (continued)

\begin{tabular}{|c|c|}
\hline Rank & Author \\
\hline 227 & Vouk, Mladen Alan* \\
\hline 228 & Jüttler, Bert \\
\hline 229 & Fuchs, Henry \\
\hline 230 & Odersky, Martin* \\
\hline 231 & Orso, Alessandro* \\
\hline 232 & Palsberg, Jens* \\
\hline 233 & Chen, Tsong Yueh* \\
\hline 234 & Magee, Jeff* \\
\hline 235 & Wei, Li Yi* \\
\hline 236 & Greenberg, Donald P. \\
\hline 237 & Walker, David* \\
\hline 238 & Prechelt, Lutz* \\
\hline 239 & Cordy, James R.* \\
\hline 240 & Harper, Robert* \\
\hline 241 & Tai, Kuo Chung* \\
\hline 242 & Rieser, John J. \\
\hline 243 & Franke, R. \\
\hline 244 & Jones, Simon Peyton* \\
\hline 245 & Hodges, Larry F.* \\
\hline 246 & Hölzle, Urs* \\
\hline 247 & LaViola, Joseph J.* \\
\hline 248 & Steed, Anthony \\
\hline 249 & Jackson, Michael* \\
\hline 250 & Hamann, Bernd \\
\hline 251 & Magnenat-Thalmann, Nadia* \\
\hline 252 & Lécuyer, Anatole* \\
\hline 253 & Wand, Mitchell* \\
\hline 254 & France, Robert B.* \\
\hline 255 & Rothermel, Gregg* \\
\hline 256 & Griswold, William G.* \\
\hline 257 & Eick, Stephen G.* \\
\hline 258 & Mei, Hong* \\
\hline 259 & Whittle, Jon* \\
\hline 260 & Kazman, Rick* \\
\hline 261 & Marcus, Andrian* \\
\hline 262 & Ko, Andrew J. \\
\hline 263 & Petersen, Kai* \\
\hline 264 & Ungar, David* \\
\hline 265 & Sneed, Harry M.* \\
\hline 266 & Pascucci, Valerio \\
\hline 267 & Knight, John* \\
\hline 268 & Rajlich, Václav* \\
\hline 269 & Perry, Dewayne E.* \\
\hline 270 & Dobkin, David P. \\
\hline 271 & Di Penta, Massimiliano* \\
\hline
\end{tabular}


Table 8 (continued)

\begin{tabular}{|c|c|}
\hline Rank & Author \\
\hline 272 & Lewis, J. P. \\
\hline 273 & Poshyvanyk, Denys* \\
\hline 274 & Murugesan, San* \\
\hline 275 & Ducasse, Stéphane* \\
\hline 276 & Sjoberg, Dag I.K.* \\
\hline 277 & Lee, Seungyong \\
\hline 278 & Weiskopf, Daniel \\
\hline 279 & DeRose, Tony \\
\hline 280 & Mendes, Emilia* \\
\hline 281 & Lämmel, Ralf* \\
\hline 282 & Schneidewind, N. F.* \\
\hline 283 & Grundy, John* \\
\hline 284 & Dingsøyr, Torgeir* \\
\hline 285 & Fuggetta, Alfonso* \\
\hline 286 & Chang, Shi Kuo* \\
\hline 287 & Nierstrasz, Oscar* \\
\hline 288 & Leung, Hareton* \\
\hline 289 & Lethbridge, Timothy C.* \\
\hline 290 & Shirley, Peter \\
\hline 291 & Wong, W. Eric* \\
\hline 292 & Boehm, Hans J.* \\
\hline 293 & Wald, Ingo \\
\hline 294 & Sewell, Peter* \\
\hline 295 & Osterweil, Leon J.* \\
\hline 296 & Corbett, James C.* \\
\hline 297 & Clarke, Lori A.* \\
\hline 298 & Snyder, John \\
\hline 299 & Ellis, Stephen R. \\
\hline 300 & Peters, Jörg \\
\hline 301 & Adve, Vikram* \\
\hline 302 & Bird, Christian* \\
\hline 303 & Apel, Sven* \\
\hline 304 & Brun, Yuriy* \\
\hline 305 & Nishita, Tomoyuki \\
\hline 306 & German, Daniel M.* \\
\hline 307 & Woodside, Murray* \\
\hline 308 & Goldman, Ron* \\
\hline 309 & Grossman, Dan* \\
\hline 310 & Fisher, Danyel \\
\hline 311 & Reinhard, Erik \\
\hline 312 & Bieman, James M.* \\
\hline 313 & Guo, Philip J. \\
\hline 314 & Peyton Jones, S. I.M.O.N.* \\
\hline 315 & Pauly, Mark \\
\hline 316 & Ramalingam, G.* \\
\hline
\end{tabular}


Table 8 (continued)

\begin{tabular}{|c|c|}
\hline Rank & Author \\
\hline 317 & Potts, Colin* \\
\hline 318 & Miller, James \\
\hline 319 & Damian, Daniela* \\
\hline 320 & Laprie, J. C.* \\
\hline 321 & Ruhe, Guenther* \\
\hline 322 & Hughes, John F.* \\
\hline 323 & Pierce, Benjamin C.* \\
\hline 324 & Cooper, Keith* \\
\hline 325 & Ashikhmin, Michael \\
\hline 326 & Petrank, Erez* \\
\hline 327 & Debray, Saumya* \\
\hline 328 & Burnett, Margaret* \\
\hline 329 & Cugola, Gianpaolo \\
\hline 330 & Deussen, Oliver \\
\hline 331 & Van de Panne, Michiel \\
\hline 332 & Babar, Muhammad Ali* \\
\hline 333 & Shen, Han Wei \\
\hline 334 & Emmerich, Wolfgang* \\
\hline 335 & Ghezzi, Carlo* \\
\hline 336 & Martin, Ralph R. \\
\hline 337 & Ammann, Paul* \\
\hline 338 & Gomaa, Hassan* \\
\hline 339 & Visser, Willem* \\
\hline 340 & Wirth, N.* \\
\hline 341 & Qadeer, Shaz* \\
\hline 342 & Matusik, Wojciech \\
\hline 343 & Agrawala, Maneesh \\
\hline 344 & McCabe, Thomas J.* \\
\hline 345 & Bodden, Eric* \\
\hline 346 & Catmull, Edwin \\
\hline 347 & O'Brien, James F. \\
\hline 348 & Lin, Ming \\
\hline 349 & Shull, Forrest* \\
\hline 350 & Ribarsky, William \\
\hline 351 & Fraser, Gordon* \\
\hline 352 & Kacker, Raghu N.* \\
\hline 353 & Bacon, David F.* \\
\hline 354 & DeLine, Robert* \\
\hline 355 & Zhu, Hong \\
\hline 356 & Guo, Baining \\
\hline 357 & Cook, William R.* \\
\hline 358 & Chilimbi, Trishul \\
\hline 359 & Laidlaw, David H. \\
\hline 360 & Zhang, Hongyu \\
\hline 361 & Mezini, Mira* \\
\hline
\end{tabular}


Table 8 (continued)

\begin{tabular}{|c|c|}
\hline Rank & Author \\
\hline 362 & Kazhdan, Misha \\
\hline 363 & Thomas, Bruce H. \\
\hline 364 & Niazi, Mahmood* \\
\hline 365 & Kautz, Jan \\
\hline 366 & Regehr, John* \\
\hline 367 & Zhou, Kun \\
\hline 368 & Benton, Nick* \\
\hline 369 & Singh, Karan \\
\hline 370 & Guéhéneuc, Yann Gaël* \\
\hline 371 & Amarasinghe, Saman* \\
\hline 372 & Hauser, Helwig \\
\hline 373 & Davis, Alan M.* \\
\hline 374 & Hudak, Paul* \\
\hline 375 & Magnor, Marcus \\
\hline 376 & Cignoni, Paolo \\
\hline 377 & Aldrich, Jonathan* \\
\hline 378 & Andrienko, Gennady \\
\hline 379 & Sheard, Tim* \\
\hline 380 & Malaiya, Yashwant K.* \\
\hline 381 & Yau, Stephen S.* \\
\hline 382 & Bultan, Tevfik* \\
\hline 383 & Lavoué, Guillaume \\
\hline 384 & Loop, Charles \\
\hline 385 & Gokhale, Swapna* \\
\hline 386 & Gorton, Ian* \\
\hline 387 & Dwyer, Matthew B.* \\
\hline 388 & Musa, John D.* \\
\hline 389 & Grunske, Lars* \\
\hline 390 & Mitra, Niloy J. \\
\hline 391 & Ostromoukhov, V. \\
\hline 392 & Firesmith, Donald G.* \\
\hline 393 & Interrante, Victoria \\
\hline 394 & Marchesi, M.* \\
\hline 395 & Rountev, Atanas \\
\hline 396 & Aliaga, Daniel G. \\
\hline 397 & Diehl, Stephan* \\
\hline 398 & Abran, Alain* \\
\hline 399 & Moss, J. Eliot B.* \\
\hline 400 & Parker, Steven \\
\hline 401 & Notkin, David* \\
\hline 402 & Lee, Jehee \\
\hline 403 & Lischinski, Dani \\
\hline 404 & Seidman, Stephen* \\
\hline 405 & Smaragdakis, Yannis* \\
\hline 406 & Van Der Hoek, Andre* \\
\hline
\end{tabular}


Table 8 (continued)

\begin{tabular}{|c|c|}
\hline Rank & Author \\
\hline 407 & Miller, Gavin S.P. \\
\hline 408 & Glinz, Martin* \\
\hline 409 & Frankl, Phyllis* \\
\hline 410 & Dykes, Jason \\
\hline 411 & Hinze, Ralf* \\
\hline 412 & Meijer, Erik* \\
\hline 413 & Lopes, Cristina V.* \\
\hline 414 & Hindle, Abram* \\
\hline 415 & Decarlo, Doug \\
\hline 416 & Ebert, David S. \\
\hline 417 & Goel, Amrit L.* \\
\hline 418 & Heitmeyer, Constance* \\
\hline 419 & Sillion, Francois \\
\hline 420 & Noble, James* \\
\hline 421 & Franz, Michael* \\
\hline 422 & $\mathrm{Xi}$, Hongwei* \\
\hline 423 & Yahav, Eran* \\
\hline 424 & Ferwerda, James \\
\hline 425 & Yoo, Shin* \\
\hline 426 & Consel, Charles* \\
\hline 427 & De Floriani, Leila \\
\hline 428 & Jézéquel, Jean Marc* \\
\hline 429 & Wagner, Stefan* \\
\hline 430 & Lo, David* \\
\hline 431 & Franch, Xavier* \\
\hline 432 & Lutz, Robyn* \\
\hline 433 & Schmid, Klaus* \\
\hline 434 & Telea, Alexandru C.* \\
\hline 435 & Biffl, Stefan* \\
\hline 436 & Patané, Giuseppe \\
\hline 437 & Kunii, Tosiyasu L.* \\
\hline 438 & Garousi, Vahid* \\
\hline 439 & Zdun, Uwe* \\
\hline 440 & Kim, Kane* \\
\hline 441 & Roy, Chanchal K.* \\
\hline
\end{tabular}

\section{Complete list of top-listed software engineering researchers}

The following table provides the list of top researchers identified for software engineering.

Funding Open access funding provided by Blekinge Institute of Technology.

Open Access This article is licensed under a Creative Commons Attribution 4.0 International License, which permits use, sharing, adaptation, distribution and reproduction in any medium or format, as long as you give appropriate credit to the original author(s) and the source, provide a link to the Creative Commons 
licence, and indicate if changes were made. The images or other third party material in this article are included in the article's Creative Commons licence, unless indicated otherwise in a credit line to the material. If material is not included in the article's Creative Commons licence and your intended use is not permitted by statutory regulation or exceeds the permitted use, you will need to obtain permission directly from the copyright holder. To view a copy of this licence, visit http://creativecommons.org/licenses/by/4.0/.

\section{References}

Baerlocher, M. O., Newton, M., Gautam, T., Tomlinson, G., \& Detsky, A. S. (2007). The meaning of author order in medical research. Journal of Investigative Medicine, 55(4), 174-180.

Bin Ali N., Edison H., \& Torkar R. (2020). The impact of a proposal for innovation measurement in the software industry. In: Proceedings of the 14th ACM / IEEE International Symposium on Empirical Software Engineering and Measurement (ESEM), pp. 1-6, Bari Italy, ACM.

Bornmann, L., \& Daniel, H. D. (2008). What do citation counts measure? A review of studies on citing behavior. Journal of Documentation, 64(1), 45-80.

Croux, Christophe, \& Dehon, Catherine. (2010). Influence functions of the spearman and kendall correlation measures. Statistical Methods \& Applications, 19(4), 497-515.

Fernandes, João. M. (2014). Authorship trends in software engineering. Scientometrics, 101(1), $257-271$.

Fernandes, João. M., \& Monteiro, Miguel P. (2017). Evolution in the number of authors of computer science publications. Scientometrics, 110(2), 529-539.

Garousi V., \& Fernandes J.M. (2016). Highly-cited papers in software engineering: The top-100. p. 21: Information and Software Technology.

Garousi, V., \& Fernandes, J. M. (2017). Quantity versus impact of software engineering papers: A quantitative study. Scientometrics, 112(2), 963-1006.

Glass, R. L. (1994). An assessment of systems and software engineering scholars and institutions. The Journal of Systems and Software, 27(1), 63-67.

Glass, R. L. (1995). Editor's corner an assessment of systems and software engineering scholars and institutions, 1993 and 1994. Journal of System and Software, 31(1), 3-6.

Glass, Robert L. (1996). An assessment of systems and software engineering scholars and institutions (1993-1995). Journal of System and Software, 35(1), 85-89.

Glass, R. L. (1998). An assessment of systems and software engineering scholars and institutions (19931997). Journal of System and Software, 43(1), 59-64.

Glass, R. L. (1999). An assessment of systems and software engineering scholars and institutions (19941998). Journal of System and Software, 49(1), 81-86.

Glass, Robert L. (2000). An assessment of systems and software engineering scholars and institutions (1995-1999). Journal of System and Software, 54(1), 77-82.

Glass, Robert L., \& Chen, Tsong Yueh. (2001). An assessment of systems and software engineering scholars and institutions (1996-2000). Journal of System and Software, 59(1), 107-113.

Glass, Robert L., \& Chen, Tsong Yueh. (2002). An assessment of systems and software engineering scholars and institutions (1997-2001). Journal of System and Software, 64(1), 79-86.

Glass, R. L., \& Chen, T. Y. (2003). An assessment of systems and software engineering scholars and institutions (1998-2002). Journal of System and Software, 68(1), 77-84.

Glass, Robert L., \& Chen, Tsong Yueh. (2005). An assessment of systems and software engineering scholars and institutions (1999-2003). Journal of System and Software, 76(1), 91-97.

Hagen, Nils T. (2014). Counting and comparing publication output with and without equalizing and inflationary bias. Journal of Informetrics, 8(2), 310-317.

Ioannidis, J. P. A., Boyack, K. W., \& Baas, J. (2020). Updated science-wide author databases of standardized citation indicators. PLOS Biology, 18(10), 1-3, 10.

Ioannidis, John P.A.., Klavans, Richard, \& Boyack, Kevin W. (2016). Multiple citation indicators and their composite across scientific disciplines. PLOS Biology, 14(7), e1002501.

Karanatsiou, D., Li, Y., Arvanitou, E. M., Misirlis, N., \& Wong, W. E. (2019). A bibliometric assessment of software engineering scholars and institutions (2010-2017). Journal of Systems and Software, 147, 246-261.

Ley, Michael. (2009). Dblp: Some lessons learned. Proceedings of the VLDB Endowment, 2(2), 1493-1500. 
Mathew, G., Agrawal, A., \& Menzies, T. (2019). Finding trends in software research. IEEE Transactions on Software Engineering. https://doi.org/10.1109/TSE.2018.2870388

Molléri, J. S., Petersen, K., \& Mendes, E. (2018). Towards understanding the relation between citations and research quality in software engineering studies. Scientometrics, 117(3), 1453-1478.

Pendlebury, D. A., \& Adams, J. (2012). Comments on a critique of the thomson reuters journal impact factor. Scientometrics, 92(2), 395-401.

Poulding, S., Petersen K., Feldt R., \& Garousi, V. (2015). In: Using citation behavior to rethink academic impact in software engineering.

Schreiber, Michael. (2008). A modification of the h-index: The hm-index accounts for multi-authored manuscripts. Journal of Informetrics, 2(3), 211-216.

Teufel, S., Siddharthan, A., \& Tidhar, D. (2006). An annotation scheme for citation function. Sydney, Australia: Association for Computational Linguistics. pp. 80.

Tse, T. H., Chen, T. Y., \& Glass, R. L. (2006). An assessment of systems and software engineering scholars and institutions (2000-2004). Journal of System and Software, 79(6), 816-819.

Wong, W. E., Tse, T. H., Glass, R. L., Basili, V. R., \& Chen, T. Y. (2008). An assessment of systems and software engineering scholars and institutions (2001-2005). Journal of System and Software, 81(6), 1059-1062.

Wong, W. E., Tse, T. H., Glass, R. L., Basili, V. R., \& Chen, T. Y. (2009). An assessment of systems and software engineering scholars and institutions (2002-2006). Journal of System and Software, 82(8), $1370-1373$.

Wong, W. E., Tse, T. H., Glass, R. L., Basili, V. R., \& Chen, T. Y. (2011). An assessment of systems and software engineering scholars and institutions (2003-2007 and 2004-2008). Journal of Systems and Software, 84(1), 162-168. 